\title{
On the Quantization of the Higher Spin Fields
}

\author{
J.W.Wagenaar and T.A.Rijken
}

April 4, 2022

\begin{abstract}
In this article we quantize (massive) higher spin $(1 \leq j \leq 2)$ fields by means of Dirac's Constrained Hamilton procedure both in the situation were they are totally free and were they are coupled to (an) auxiliary field(s). A full constraint analysis and quantization is presented by determining and discussing all constraints and Lagrange multipliers and by giving all equal times (anti) commutation relations. Also we construct the relevant propagators. In the free case we obtain the well-known propagators and show that they are not covariant, which is also well known. In the coupled case we do obtain covariant propagators (in the spin-3/2 case this requires $b=0$ ) and show that they have a smooth massless limit connecting perfectly to the massless case (with auxiliary fields). We notice that in our system of the spin- $3 / 2$ and spin- 2 case the massive propagators coupled to conserved currents only have a smooth limit to the pure massless spin-propagator, when there are ghosts in the massive case.
\end{abstract}

\section{Introduction}

This article is about the quantization of higher spin $(1 \leq j \leq 2)$ fields and their propagators. Besides the interest in their own, the physical interest in these various fields comes from very different areas in (high energy) physics. The massive spin-1 field is extremely important in the electro-weak part of the Standard Model and in phenomenological One-Boson-Exchange (OBE) models. Needless to mention the physical interest in the photon.

As far as the spin-3/2 field is concerned, ever since the pioneering work of [1] and [2 it has been considered by many authors for several reasons. The spin- $3 / 2$ field plays a significant role in low energy hadron scattering, where it appears as a resonance. Also in supergravity (for a review see [3]) and superstring theory the spin-3/2 field plays an important role, since it appears in these theories as a massless gravitino. Besides the role it plays in the tensor-force in OBE-models the spin-2 field mainly appears in (super-) gravity and string theories as the massless graviton.

The quantization of such fields can roughly be divided in three areas: free field quantization, the quantization of the system where it is coupled to (an) auxiliary field(s) and the quantization of an interacting field. The latter area in the spin-3/2 case is known to have problems and inconsistencies (see for instance [4, 5] and 6]). Although very interesting, in this article we will focus our attention on the first two areas.

In section 2 we start with the quantization of the massive, free fields. We do this for all spin cases $(j=1,3 / 2,2)$ at the same time using Dirac's prescription 7 . The inclusion of the spin- 1 field case is merely meant to demonstrate Dirac's procedure in a simple case and to have a complete description of higher spin field quantization.

The free spin-3/2 field quantization is in the same line as in references $8,9,10,11$. In 8 the massless free spin-3/2 field was quantized in the transverse gauge. The authors of [9, 10, quantize the massive free theory, which is also what we do. We will follow Dirac's prescription straightforwardly by first determining all Lagrange multipliers and constraints. Afterwards the Dirac bracket (Db) is introduced and we calculate the equal time anti commutation (ETAC) relations among all components of the field. In both 9] and 10, 11 the step to the Dirac bracket is made earlier, without determining all Lagrange multipliers and constraints. In 9 it is mentioned that this involves "technical difficulties and much 
labor" and in 10, 11 the focus is on the number of constraints and therefore not so much on their specific forms. As a result [9] and [10, 11] both calculate only the ETAC relations between the spatial components of the spin-3/2 field, whereas we obtain them all.

A Dirac constraint analysis of the free spin-2 field can be found for instance in [12, 13, 14. In these references the massless ([12, 13]) case and massive (14]) case is considered. We stress, however, that our description of the quantization not only differs from [14 in the sense that the nature of one of the obtained constraints is different, which we will discuss below, also we obtain all constraints and Lagrange multipliers by applying Dirac's procedure straightforward. We present a full analysis of the constrained system. After introducing the Dirac bracket $(\mathrm{Db})$ we give all equal time commutation (ETC) relations between the various components of the spin-2 field.

Having quantized the free theories properly we make use of a free field expansion identity and with these ingredients we obtain the propagators. We notice that they are not explicitly covariant, as is mentioned for instance in [15] for general cases $j \geq 1$.

To cure this problem we are inspired by [16] and allow for auxiliary fields in the free Lagrangian in section 3 To be more specific we couple gauge conditions of the massless cases to auxiliary fields and also allow for mass terms of these auxiliary fields, with which free (gauge) parameters are introduced. As in for instance [16], we obtain a covariant vector field propagator, independently of the choice of the parameter.

In the spin- $3 / 2$ case several systems of a spin- $3 / 2$ field coupled to auxiliary fields are considered in [17, 18, 19. In [18, 19, are for several of such systems four dimensional commutation relations obtained. In the only massive case which the authors of [19] consider, two auxiliary fields are introduced to couple (indirectly) to the constraint equations 1 of a spin-3/2 field. The authors of 17 use the Lagrange multiplier $\square$ method, where this multiplier is coupled to the covariant gauge condition of the massless spin-3/2 field in the Rarita-Schwinger (RS) framework (to be defined below). They notice that the Lagrange multiplier has to be a spinor and in this sense it can also be viewed as an auxiliary field. We follow the same line by coupling our auxiliary field to the above mentioned gauge condition. In 17. the quantization is performed outside the RS framework in order to circumvent the appearance of singularities. We remain within the RS framework and deal with these singularities relying on Dirac's method. Therefore we stay in line with the considerations of section 2 A covariant propagator is obtained for one specific choice of the parameter $(b=0)$. This propagator is the same as the one obtained in [17. We notice that also in [20] a covariant propagator is obtained, but these authors make use of two spin- $1 / 2$ fields.

Coupled systems of spin-2 and auxiliary fields were for various reasons considered in for instance 21, 22, 23, 24, 25. In 22, an auxiliary boson field is coupled to the "De Donder" gauge condition in the Lagrangian which also contains Faddeev-Popov ghosts. In 23] an auxiliary field is coupled to the divergence of the tensor field in such a way that the auxiliary field can be viewed as a Lagrange multiplier. These authors mention that if an other auxiliary field is introduced, coupled to the trace of the tensor field in order to get the other spin-2 condition, four dimensional commutation relations for the tensor field can not be written down. We present a description in which this is possible relying on Dirac's procedure. Also in the tensor field case we obtain a covariant propagator, independently of the choice of the parameter.

Having obtained all the various covariant propagators we discuss several choices of the parameters (if possible) and the massless limits of these propagators. We show that the propagators do not only have a smooth massless limit but that they also connect to the ones obtained in the massless case (including (an) auxiliary field(s)).

When coupled to conserved currents we see that it is possible to obtain the correct massless spin- $j$ propagators carrying only the helicities $\lambda= \pm j_{z}$. This does not require a choice of the parameter in the spin- 1 case, but in the spin- $3 / 2$ and in the spin- 2 case we have to make the choices $b=03$ and $c= \pm \infty$. As far as these last two cases is concerned, it is a different situation then taking the massive propagator,

\footnotetext{
${ }^{1} i \partial \psi=0$ is a constraint in the sense that it reduces the number of degrees of freedom of a general $\psi_{\mu}$ field. It is not a constraint in the sense of Dirac, since it is a dynamical equation.

${ }^{2}$ These Lagrange multipliers are the ones used in the original sense and are therefore different then the ones used in Dirac's formalism.

${ }^{3}$ This choice we already made in order to obtain a covariant propagator.
} 
couple it to conserved currents and putting the mass to zero as noticed in 26] and 27, respectively. A discussion on the latter matter in (anti)-de Sitter spaces can be found in [28, 29, 30. We stress however, that in the spin-3/2 and the spin- 2 case this limit is only smooth if the massive propagator contains ghosts.

\section{Free Fields}

As mentioned in the introduction we deal with the free theories in this section. We start in section 2.1 with the Lagrangians and the equations of motion that can be deduced from them. We explicitly quantize the theories in section 2.2 and calculate the propagators in section 2.3

\subsection{Equations of Motion}

As a starting point we take the Lagrangian for free, massive fields $(j=1,3 / 2,2)$. In case of the spin-3/2 there is, according to [11, 31, 32, 33, 34, a class of Lagrangians describing the particularities of a spin-3/2 field. Also in the spin-2 case several authors ([23, 35, 36, 37]) describe a class of Lagrangians (with one or more free parameters) which give the correct Euler-Lagrange equations for a spin-2 field. By taking this spin-2 field to be real and symmetric from the outset only one parameter remains

$$
\begin{aligned}
\mathcal{L}_{1}= & -\frac{1}{2}\left(\partial_{\mu} A_{\nu} \partial^{\mu} A^{\nu}-\partial_{\mu} A_{\nu} \partial^{\nu} A^{\mu}\right)+\frac{1}{2} M_{1}^{2} A^{\mu} A_{\mu}, \\
\mathcal{L}_{3 / 2, A}= & \bar{\psi}^{\mu}\left[\left(i \not \partial-M_{3 / 2}\right) g_{\mu \nu}+A\left(\gamma_{\mu} i \partial_{\nu}+\gamma_{\nu} i \partial_{\mu}\right)+B \gamma_{\mu} i \not \partial \gamma_{\nu}+C M_{3 / 2} \gamma_{\mu} \gamma_{\nu}\right] \psi^{\nu}, \\
\mathcal{L}_{2, A}= & \frac{1}{4} \partial^{\alpha} h^{\mu \nu} \partial_{\alpha} h_{\mu \nu}-\frac{1}{2} \partial_{\mu} h^{\mu \nu} \partial^{\alpha} h_{\alpha \nu}-\frac{1}{4} B \partial_{\nu} h_{\beta}^{\beta} \partial^{\nu} h_{\alpha}^{\alpha}-\frac{1}{2} A \partial_{\alpha} h^{\alpha \beta} \partial_{\beta} h_{\nu}^{\nu} \\
& -\frac{1}{4} M_{2}^{2} h^{\mu \nu} h_{\mu \nu}+\frac{1}{4} C M_{2}^{2} h_{\mu}^{\mu} h_{\nu}^{\nu},
\end{aligned}
$$

where $B=\frac{1}{2}\left(3 A^{2}+2 A+1\right), C=3 A^{2}+3 A+1$ and $A \neq-\frac{1}{2}$, but arbitrary otherwise. We improperly 4 refer to (1b) as the RS case.

Since we do not need to be so general we choose $A=-1$ and end-up with a particular spin- $3 / 2$ Lagrangian also used in [3, 8, 9, 10, 11, 19] and in case of the spin-2 field we get the well-know FierzPauli Lagrangian [1] also used in for instance [38, 39, 40]

$$
\begin{aligned}
\mathcal{L}_{3 / 2}= & -\frac{1}{2} \epsilon^{\mu \nu \rho \sigma} \bar{\psi}_{\mu} \gamma_{5} \gamma_{\rho}\left(\partial_{\sigma} \psi_{\nu}\right)+\frac{1}{2} \epsilon^{\mu \nu \rho \sigma}\left(\partial_{\sigma} \bar{\psi}_{\mu}\right) \gamma_{5} \gamma_{\rho} \psi_{\nu}-M_{3 / 2} \bar{\psi}_{\mu} \sigma^{\mu \nu} \psi_{\nu}, \\
\mathcal{L}_{2}= & \frac{1}{4} \partial^{\alpha} h^{\mu \nu} \partial_{\alpha} h_{\mu \nu}-\frac{1}{2} \partial_{\mu} h^{\mu \nu} \partial^{\alpha} h_{\alpha \nu}-\frac{1}{4} \partial_{\nu} h_{\beta}^{\beta} \partial^{\nu} h_{\alpha}^{\alpha}+\frac{1}{2} \partial_{\alpha} h^{\alpha \beta} \partial_{\beta} h_{\nu}^{\nu} \\
& -\frac{1}{4} M_{2}^{2} h^{\mu \nu} h_{\mu \nu}+\frac{1}{4} M_{2}^{2} h_{\mu}^{\mu} h_{\nu}^{\nu} .
\end{aligned}
$$

Although we have picked particular Lagrangians we can always go back to the general case by redefining the fields in the following sense

$$
\begin{array}{ll}
\psi_{\mu}^{\prime}=O_{\mu}^{\alpha}(A) \psi_{\alpha}, & O_{\mu}^{\alpha}(A)=g_{\mu}^{\alpha}-\frac{A+1}{2} \gamma_{\mu} \gamma^{\alpha}, \\
h_{\mu \nu}^{\prime}=O_{\mu \nu}^{\alpha \beta}(A) h_{\alpha \beta}, & O_{\mu \nu}^{\alpha \beta}(A)=\frac{1}{2}\left(g_{\mu}^{\alpha} g_{\nu}^{\beta}+g_{\mu}^{\beta} g_{\nu}^{\alpha}-(A+1) g_{\mu \nu} g^{\alpha \beta}\right) .
\end{array}
$$

The transformation in the first line of (3) was also mentioned in [11. Requiring that the transformation matrices in (3) are non-singular $(\operatorname{det} O \neq 0)$ gives again the constraint $A \neq-\frac{1}{2}$.

The Euler-Lagrange equations following from the free field Lagrangians lead to the correct equations of motion (EoM)

$$
\begin{aligned}
\left(\square+M_{1}^{2}\right) A^{\mu}=0 \quad, & \partial \cdot A=0 \quad, & \\
\left(i \not \partial-M_{3 / 2}\right) \psi_{\mu}=0 \quad, & \gamma \cdot \psi=0 \quad, & i \partial \cdot \psi=0, \\
\left(\square+M_{2}^{2}\right) h^{\mu \nu}=0 \quad, & \partial_{\mu} h^{\mu \nu}=0 \quad, & h_{\mu}^{\mu}=0 .
\end{aligned}
$$

\footnotetext{
${ }^{4}$ Although the authors of [2] mention a general class, they expose one specific Lagrangian which would correspond to the choice $A=-\frac{1}{3}$
} 
The massless versions of the Lagrangians $\mathcal{L}_{1}, \mathcal{L}_{3 / 2}$ and $\mathcal{L}_{2}$ 占 exhibit a gauge freedom: they are invariant under the transformations $A^{\mu} \rightarrow A^{\mu \prime}=A^{\mu}+\partial^{\mu} \Lambda, \psi_{\mu} \rightarrow \psi_{\mu}^{\prime}=\psi_{\mu}+\partial_{\mu} \epsilon$ and $h^{\mu \nu} \rightarrow h^{\mu \nu \prime}=h^{\mu \nu}+\partial^{\mu} \eta^{\nu}+$ $\partial^{\nu} \eta^{\mu}$ as well as $h^{\mu \nu} \rightarrow h^{\mu \nu \prime}=h^{\mu \nu}+\partial^{\mu} \partial^{\nu} \Lambda$, respectively. Here, $\Lambda, \epsilon$ and $\eta^{\mu}$ are scalar, spinor and vector fields, respectively.

In the spin- 1 case a popular gauge is the Lorentz gauge $\partial \cdot A=0$. Imposing this gauge conditions automatically ensures the EoM $\square A^{\mu}=0$ and puts the constraint $\square \Lambda=0$. This last constraint is used to eliminate the residual helicity state $\lambda=0$.

A popular gauge in the spin-3/2 case is the covariant gauge $\gamma \cdot \psi=0$, which causes similar effects, namely the correct EoM $i \not \partial \psi=0$ and $i \partial \cdot \psi=0$ and the constraint $i \not \partial \epsilon$. Since the $\epsilon$-field is a free spinor, it is used to transform away the helicity states $\lambda= \pm 1 / 2$ of the free $\psi_{\mu}$ field.

Since the spin-2 Lagrangian has two symmetries, two gauge conditions need to be imposed. The gauge conditions $h_{\alpha}^{\alpha}=0$ and $\partial_{\alpha} h^{\alpha \beta}=0$ give the correct EoM. From the effects these gauge conditions have on the auxiliary fields $\left(\square \eta^{\mu}=0, \partial \cdot \eta=0\right.$ and $\left.\square \Lambda=0\right)$ we see that these equations describe a massless spin- 1 field and a massless spin-0 field. Therefore these fields can be used to ensure that the tensor field $h^{\mu \nu}$ only has $\lambda= \pm 2$ helicity states.

In our case the mass terms in the Lagrangian break the gauge symmetry. Although, the correct EoM (4) are obtained the freedom in the choice of the field can not be exploited to transform away helicity states. Therefore, the massive fields contain all helicity states, as is of course well known.

\subsection{Quantization}

For the quantization of our systems we use Dirac's Hamilton method for constrained systems [7]. In case of the (real) vector and tensor fields the accompanying canonical momenta are defined in the usual way. Since we use complex fields in case of the spin-3/2 field we consider $\psi_{\mu}$ and $\psi_{\mu}^{\dagger}$ as independent fields being elements of a Grassmann algebra. For the definition of the accompanying canonical momenta we rely on [42. Although, the authors of 42 use spin-1/2 fields, the prescription for the canonical momenta does not change. The canonical momenta are defined as

$$
\pi_{a}^{\nu}=\frac{\partial^{r} \mathcal{L}}{\partial \dot{\psi}_{a, \nu}} \quad, \quad \pi_{a}^{\nu \ddagger}=\frac{\partial^{r} \mathcal{L}}{\partial \dot{\psi}_{a, \nu}^{*}},
$$

where $r$ means that the differentiation is performed from right to left. We use the $\ddagger$-notation to distinguish the canonical momentum coming from the complex conjugate field from the one coming form the original field, since they need not (and in fact will not) be the same. 2b)

Using this prescription (5) we obtain the canonical momenta from our Lagrangians (1a), (2a) and

$$
\begin{array}{ll}
\pi_{1}^{0}=0, & \pi_{1}^{n}=-\dot{A}^{n}+\partial^{n} A^{0}, \\
& \pi_{3 / 2}^{0}{ }^{\ddagger}=0, \\
\pi_{3 / 2}^{0}=0, & \pi_{3 / 2}^{n}{ }^{\ddagger}=\frac{i}{2} \sigma^{n k} \psi_{k}, \\
\pi_{3 / 2}^{n}=\frac{i}{2} \psi_{k}^{\dagger} \sigma^{k n}, & \pi_{2}^{0 m}=-\partial_{n} h^{n m}+\frac{1}{2} \partial^{m} h^{00} \\
& +\frac{1}{2} \partial^{m} h_{n}^{n},
\end{array}
$$

from which the velocities can be deduced

$$
\begin{aligned}
\dot{A}^{n} & =-\pi_{1}^{n}+\partial^{n} A^{0}, \\
\dot{h}^{n m} & =2 \pi_{2}^{n m}-g^{n m} \pi_{2 k}^{k}+\frac{1}{2} g^{n m} \partial_{k} h^{k 0}, \\
\dot{h}_{k}^{k} & =-\pi_{2 k}^{k}+\frac{3}{2} \partial_{k} h^{k 0},
\end{aligned}
$$

${ }^{5}$ The massless version of (2b) is the linearized Einstein-Hilbert Lagrangian discussed in many textbooks as for instance 41 
and the primary constraint equations

$$
\begin{array}{ll}
\theta_{1}^{0}=\pi_{1}^{0}, & \\
\theta_{3 / 2}^{0}=\pi_{3 / 2}^{0}, & \theta_{3 / 2}^{0}{ }^{\ddagger}=\pi_{3 / 2}^{0}{ }^{\ddagger}, \\
\theta_{3 / 2}^{n}=\pi_{3 / 2}^{n}-\frac{i}{2} \psi_{k}^{\dagger} \sigma^{k n}, & \theta_{3 / 2}^{n}=\pi_{3 / 2}^{n}-\frac{i}{2} \sigma^{n k} \psi_{k}, \\
\theta_{2}^{00}=\pi_{2}^{00}+\frac{1}{2} \partial_{n} h^{n 0}, & \theta_{2}^{0 m}=\pi_{2}^{0 m}+\partial_{n} h^{n m}-\frac{1}{2} \partial^{m} h^{00}-\frac{1}{2} \partial^{m} h_{n}^{n} .
\end{array}
$$

They vanish in the weak sense, to which we will come back below.

If we want these constraints to remain zero we impose the time derivative of these constraints to be zero. We find it most easily to define the time derivative via the Poisson bracket $(\mathrm{Pb}) \dot{\theta}=\{\theta, H\}_{P}+\partial \theta / \partial t$ 6. We, therefore, need the Hamiltonians.

Dirac has shown [7] that the Hamiltonian obtained in the usual way is a weak equation 7 and does not give the correct EoM. This can be repaired by adding the primary constraints (8) to the Hamiltonian by means of Lagrange multipliers in order to make it a so-called strong equation. What we get is

$$
\begin{aligned}
H_{w}= & \int d^{3} x \mathcal{H}_{w}(x)=\int d^{3} x\left(\sum_{i} \pi_{i} \dot{q}_{i}-\mathcal{L}\right) \\
\mathcal{H}_{1, S}= & -\frac{1}{2} \pi_{1}^{n} \pi_{1, n}+\pi_{1}^{n} \partial_{n} A_{0}+\frac{1}{2} \partial_{m} A_{n} \partial^{m} A^{n}-\frac{1}{2} \partial_{m} A_{n} \partial^{n} A^{m}-\frac{1}{2} M_{1}^{2} A^{0} A_{0} \\
& -\frac{1}{2} M_{1}^{2} A^{n} A_{n}+\lambda_{1,0} \theta_{1}^{0}, \\
\mathcal{H}_{3 / 2, S}= & \frac{1}{2} \epsilon^{\mu \nu \rho k} \bar{\psi}_{\mu} \gamma_{5} \gamma_{\rho}\left(\partial_{k} \psi_{\nu}\right)-\frac{1}{2} \epsilon^{\mu \nu \rho k}\left(\partial_{k} \bar{\psi}_{\mu}\right) \gamma_{5} \gamma_{\rho} \psi_{\nu}+M_{3 / 2} \bar{\psi}_{\mu} \sigma^{\mu \nu} \psi_{\nu} \\
& +\lambda_{3 / 2,0} \theta_{3 / 2}^{0}+\lambda_{3 / 2, n} \theta_{3 / 2}^{n}+\lambda_{3 / 2,0}^{\ddagger} \theta_{3 / 2}^{0}+\lambda_{3 / 2, n}^{\ddagger} \theta_{3 / 2}^{\ddagger}{ }^{\ddagger}, \\
\mathcal{H}_{2, S}= & \pi_{2}^{n m} \pi_{2, n m}-\frac{1}{2} \pi_{2 n}^{n} \pi_{2}^{m}+\frac{1}{2} \pi_{2 n}^{n} \partial^{m} h_{m 0}-\frac{1}{2} \partial^{k} h^{n 0} \partial_{k} h_{n 0}-\frac{1}{4} \partial^{k} h^{n m} \partial_{k} h_{n m} \\
& +\frac{1}{8} \partial_{n} h^{n 0} \partial^{m} h_{m 0}+\frac{1}{2} \partial_{n} h^{n m} \partial^{k} h_{k m}+\frac{1}{2} \partial_{m} h^{00} \partial^{m} h_{n}^{n}+\frac{1}{4} \partial_{m} h_{n}^{n} \partial^{m} h_{k}^{k} \\
& -\frac{1}{2} \partial_{n} h^{n m} \partial_{m} h_{00}-\frac{1}{2} \partial_{n} h^{n m} \partial_{m} h_{k}^{k}+\frac{1}{2} M_{2}^{2} h^{n 0} h_{n 0}+\frac{1}{4} M_{2}^{2} h^{n m} h_{n m} \\
& -\frac{1}{2} M_{2}^{2} h^{00} h_{m}^{m}-\frac{1}{4} M_{2}^{2} h_{n}^{n} h_{m}^{m}+\lambda_{2,00} \theta_{2}^{00}+\lambda_{2,0 m} \theta_{2}^{0 m} \cdot
\end{aligned}
$$

For the definition of the $\mathrm{Pb}$ we rely on [8] and [42]. There, it is defined as

$$
\{E(x), F(y)\}_{P}=\left[\frac{\partial^{r} E(x)}{\partial q_{a}(x)} \frac{\partial^{l} F(y)}{\partial p^{a}(y)}-(-1)^{n_{E} n_{F}} \frac{\partial^{r} F(y)}{\partial q_{a}(y)} \frac{\partial^{l} E(x)}{\partial p^{a}(x)}\right] \delta^{3}(x-y),
$$

where $n_{E}, n_{F}$ is $0(1)$ in case $E(x), F(x)$ is even (odd). With this form of the $\mathrm{Pb}(10)$ we already anticipate that bosons satisfy commutation relations and fermions anti-commutation relations in a quantum theory.

Now, we can impose the time derivatives of the constraints (8) to be zero using (9) and (10)

$$
\begin{aligned}
\left\{\theta_{1}^{0}(x), H_{1, S}\right\}_{P} & =\partial_{n} \pi_{1}^{n}+M_{1}^{2} A^{0}=0 \equiv \Phi_{1}^{0}(x), \\
\left\{\theta_{3 / 2}^{0}(x), H_{3 / 2, S}\right\}_{P} & =\epsilon^{\mu 0 \rho k}\left(\partial_{k} \bar{\psi}_{\mu}\right) \gamma_{5} \gamma_{\rho}-M_{3 / 2} \bar{\psi}_{\mu} \sigma^{\mu 0}=0 \equiv-\Phi_{3 / 2}^{0}{ }^{\ddagger}(x), \\
\left\{\theta_{3 / 2}^{0}{ }^{\ddagger}(x), H_{3 / 2, S}\right\}_{P} & =-\epsilon^{\mu 0 \rho k} \gamma^{0} \gamma_{5} \gamma_{\rho}\left(\partial_{k} \psi_{\mu}\right)+M_{3 / 2} \gamma^{0} \sigma^{0 \mu} \psi_{\mu}=0 \equiv-\Phi_{3 / 2}^{0}(x), \\
\left\{\theta_{3 / 2}^{n}(x), H_{3 / 2, S}\right\}_{P} & =\epsilon^{\mu n \rho k}\left(\partial_{k} \bar{\psi}_{\mu}\right) \gamma_{5} \gamma_{\rho}-M_{3 / 2} \bar{\psi}_{\mu} \sigma^{\mu n}+i \lambda_{3 / 2, k}^{\ddagger} \sigma^{k n}=0, \\
\left\{\theta_{3 / 2}^{n}{ }^{\ddagger}(x), H_{3 / 2, S}\right\}_{P} & =-\epsilon^{\mu n \rho k} \gamma^{0} \gamma_{5} \gamma_{\rho}\left(\partial_{k} \psi_{\mu}\right)+M_{3 / 2} \gamma^{0} \sigma^{n \mu} \psi_{\mu}+i \sigma^{n k} \lambda_{3 / 2, k}=0,
\end{aligned}
$$

\footnotetext{
${ }^{6}$ In practice it will turn out that the constraints do not explicitly depend on time $t$

${ }^{7}$ In constructing the usual Hamiltonian explicit use can be made of the constraints, since these are also weak equations
} 


$$
\begin{aligned}
\left\{\theta_{2}^{00}(x), H_{2, S}\right\}_{P} & =\frac{1}{2}\left[\left(\partial^{k} \partial_{k}+M_{2}^{2}\right) h_{m}^{m}-\partial_{n} \partial_{m} h^{n m}\right]=0 \equiv \frac{1}{2} \Phi_{2}^{0}(x), \\
\left\{\theta_{2}^{0 m}(x), H_{2, T o t}\right\}_{P} & =2 \partial_{k} \pi_{2}^{k m}-\left(\partial^{k} \partial_{k}+M_{2}^{2}\right) h^{0 m}=0 \equiv \Phi_{2}^{m}(x) .
\end{aligned}
$$

8 In two cases (11d) and (11e) Lagrange multipliers are determined. In all other cases new, secondary, constraints are obtained. We also impose the time derivatives of these secondary constraints to be zero

$$
\begin{aligned}
\left\{\Phi_{1}^{0}(x), H_{1, S}\right\}_{P} & =M_{1}^{2}\left(\partial_{n} A^{n}+\lambda_{1}^{0}\right)=0, \\
\left\{\Phi_{3 / 2}^{0}(x), H_{3 / 2, S}\right\}_{P} & =\sigma^{n k} i \partial_{n} \lambda_{3 / 2, k}+M_{3 / 2} \gamma^{k} \lambda_{3 / 2, k}=0, \\
\left\{\Phi_{3 / 2}^{0}{ }^{\ddagger}(x), H_{3 / 2, S}\right\}_{P} & =i \partial_{k} \lambda_{3 / 2, n}^{\ddagger} \sigma^{n k}+M_{3 / 2} \lambda_{3 / 2, k}^{\ddagger} \gamma^{k}=0, \\
\left\{\Phi_{2}^{0}(x), H_{2, S}\right\}_{P} & =-2 \partial_{n} \partial_{m} \pi_{2}^{n m}-M^{2} \pi_{2 n}^{n}+\left(\partial^{k} \partial_{k}+\frac{3}{2} M_{2}^{2}\right) \partial^{n} h_{n 0}=0 \\
& \equiv-\Phi_{2}^{(1)}(x), \\
\left\{\Phi_{2}^{m}(x), H_{2, S}\right\}_{P} & =-M_{2}^{2}\left[\lambda_{2}^{0 m}+\partial_{k} h^{k m}-\partial^{m} h^{00}-\partial^{m} h_{n}^{n}\right]=0 .
\end{aligned}
$$

The first line (12a) determines the Lagrange multiplier $\lambda_{1}^{0}$. Since this was the only Lagrange multiplier in the spin- 1 case all Lagrange multipliers of this case are determined and therefore all constraints are second class.

Equation (12e) determines the Lagrange multiplier $\lambda_{2}^{0 m}$ and equation (12d) brings about yet another (tertiary) constraint. Its vanishing time derivative yields

$$
\begin{aligned}
\left\{\Phi_{2}^{(1)}(x), H_{2, S}\right\}_{P}=M_{2}^{2} & {\left[\left(2 \partial^{k} \partial_{k}+\frac{3}{2} M_{2}^{2}\right) h^{00}+\left(\frac{3}{2} \partial^{k} \partial_{k}+M_{2}^{2}\right) h_{n}^{n}\right.} \\
& \left.-\frac{3}{2} \partial_{n} \partial_{m} h^{n m}-2 \partial_{n} \lambda_{2}^{n 0}\right]=0 .
\end{aligned}
$$

We see that we have in the spin- $3 / 2$ case as well as in the spin- 2 case two equations involving the same Lagrange multipliers. In the spin-3/2 case these are (11e) and (12b) for $\lambda_{3 / 2, k}$ and (11d) and (12c) for $\lambda_{3 / 2, k}^{\ddagger}$. In the spin-2 case these are (12e) and (13) for $\lambda_{2}^{n 0}$. Combining these equations for consistency, and using $\Phi_{3 / 2}^{0}, \Phi_{3 / 2}^{0}{ }^{\ddagger}$ as well as $\Phi_{2}^{0}$ as weakly vanishing constraints, yields the last constraints

$$
\begin{aligned}
\Phi_{3 / 2}^{(1)} & =\gamma^{0} \psi_{0}+\gamma^{k} \psi_{k}, \\
\Phi_{3 / 2}^{(1) \ddagger} & =-\psi_{0}^{\dagger} \gamma^{0}+\psi_{k}^{\dagger} \gamma^{k}, \\
\Phi_{2}^{(2)} & =h_{0}^{0}+h_{n}^{n},
\end{aligned}
$$

It is important to note that these constraints are only obtained when combining other results, as describes above. This is not done in [14. Therefore these authors do not find $\Phi_{2}^{(2)}$, leaving $\theta_{2}^{00}$ as a first class constraint. Imposing vanishing time derivatives of these constraints (14a)-(14c)

$$
\begin{aligned}
\left\{\Phi_{3 / 2}^{(1)}(x), H_{3 / 2, S}\right\}_{P} & =-\gamma^{0} \lambda_{3 / 2,0}-\gamma^{k} \lambda_{3 / 2, k}=0, \\
\left\{\Phi_{3 / 2}^{(1)}(x), H_{3 / 2, S}\right\}_{P} & =\lambda_{3 / 2,0}^{\ddagger} \gamma^{0}-\lambda_{3 / 2, k}^{\ddagger} \gamma^{k}=0, \\
\left\{\Phi_{2}^{(2)}(x), H_{2, S}\right\}_{P} & =\lambda_{2}^{00}-\pi_{2 k}^{k}+\frac{3}{2} \partial_{k} h^{k 0}=0,
\end{aligned}
$$
${ }^{8}$ If $\Phi$ is a constraint, then so is $a \Phi$. The constants in front of the constraints in (11) are chosen for convenience and have no
physical meaning. 
determines the last Lagrange multipliers $\lambda_{3 / 2,0}, \lambda_{3 / 2,0}^{\ddagger}$ and $\lambda_{2}^{00}$.

In the massless spin- 1 case the vanishing of the time-derivative of $\Phi_{1}^{0}(x)$ would automatically be satisfied as can be seen from (12a). In this case $\lambda_{1}^{0}$ would not be determined which means that both constraints are first class.

We notice that in combining the equations that involve $\lambda_{3 / 2, k}\left([11 \mathrm{e}),(12 \mathrm{~b})\right.$ ) and $\lambda_{3 / 2, k}^{\ddagger}([11 \mathrm{~d})$, (12c) $)$ we obtain the constraints $\Phi_{3 / 2}^{(1)}$ and $\Phi_{3 / 2}^{(1)^{\ddagger}}$ being proportional to $M_{3 / 2}^{2}$. This means that in the massless case these equations are already consistent with each other and that $\lambda_{3 / 2,0}$ and $\lambda_{3 / 2,0}^{\ddagger}$ can not be determined leaving $\theta_{3 / 2}^{0}$ and $\theta_{3 / 2}^{0}{ }^{\ddagger}$ to be a first class constraint $(8,8$.

The situation in the massless spin-2 case is even more clear. From (12e) and (13) it is evident that the time derivatives of $\Phi_{2}^{m}$ and $\Phi_{2}^{(1)}$ will already be zero and that $\lambda_{2}^{0 k}$ can not be determined. Therefore $\Phi_{2}^{(2)}$ will not be obtained from which $\lambda_{2}^{00}$ also can not be determined, leaving $\theta_{2}^{00}$ and $\theta_{2}^{0 n}$ to be first class constraints $([12,13]) 10$

The fact that there are first class constraints (or undetermined Lagrange multipliers) in the massless cases is a reflection of the gauge symmetry. In the spin- 1 and the spin- $3 / 2$ case only one Lagrange multiplier is undetermined meaning there's only one gauge symmetry (of course the massless spin- $3 / 2$ action is also invariant under the hermitian gauge transformation, that's why $\lambda_{3 / 2, k}^{\ddagger}$ is also undetermined). In the massless spin-2 case, however, there are two Lagrange multipliers undetermined, meaning that there are two gauge symmetries as we have mentioned before.

In the massive cases all Lagrange multipliers can be determined, which means that all constraints are second class. Therefore every constraint has at least one non-vanishing $\mathrm{Pb}$ with another constraint. The complete set of constraints (primary, secondary, ... ) is

$$
\begin{aligned}
& \theta_{1}^{0}=\pi_{1}^{0}, \quad \Phi_{1}^{0}=\partial_{n} \pi_{1}^{n}+M_{1}^{2} A^{0}, \\
& \theta_{3 / 2}^{0}=\pi_{3 / 2}^{0}, \quad \theta_{3 / 2}^{0}{ }^{\ddagger}=\pi^{0^{\ddagger}}, \\
& \Phi_{3 / 2}^{(1)}=\gamma \cdot \psi, \quad \Phi_{3 / 2}^{(1) \ddagger}=-\psi_{0}^{\dagger} \gamma^{0}+\psi_{k}^{\dagger} \gamma^{k}, \\
& \theta_{3 / 2}^{n}=\pi_{3 / 2}^{n}-\frac{i}{2} \psi_{k}^{\dagger} \sigma^{k n}, \quad \theta_{3 / 2}^{n}{ }^{\ddagger}=\pi^{n \ddagger}-\frac{i}{2} \sigma^{n k} \psi_{k}, \\
& \Phi_{3 / 2}^{0}=-i \partial_{k} \sigma^{k l} \psi_{l}-M_{3 / 2} \gamma^{k} \psi_{k}, \quad \Phi_{3 / 2}^{0}{ }^{\ddagger}=-\psi_{n}^{\dagger} \sigma^{n k} i \overleftarrow{\partial_{k}}-M_{3 / 2} \psi_{k}^{\dagger} \gamma^{k} \\
& \begin{array}{ll}
\theta_{2}^{00}=\pi_{2}^{00}+\frac{1}{2} \partial_{n} h^{n 0}, & \Phi_{2}^{0}=\left(\partial^{k} \partial_{k}+M_{2}^{2}\right) h_{m}^{m}-\partial_{n} \partial_{m} h^{n m} \\
\theta_{2}^{0 m}=\pi_{2}^{0 m}+\partial_{n} h^{n m}-\frac{1}{2} \partial^{m} h^{00} & \Phi_{2}^{m}=2 \partial_{k} \pi^{k m}-\left(\partial^{k} \partial_{k}+M_{2}^{2}\right) h^{0 m},
\end{array}, \\
& -\frac{1}{2} \partial^{m} h_{n}^{n}, \quad \Phi_{2}^{(2)}=h_{0}^{0}+h_{n}^{n}, \\
& \Phi_{2}^{(1)}=2 \partial_{n} \partial_{m} \pi_{2}^{n m}+M_{2}^{2} \pi_{2 n}^{n} \\
& -\left(\partial^{k} \partial_{k}+\frac{3}{2} M_{2}^{2}\right) \partial^{n} h_{n 0} \text {, }
\end{aligned}
$$

We want to make linear combinations of constraints in order to reduce the number of non-vanishing $\mathrm{Pb}$ among these constraints. In the end we will arrive at a situation where every constraint has only one non-vanishing $\mathrm{Pb}$ with another constraint. Therefore, we make the following linear combinations

$$
\begin{aligned}
\tilde{\theta}_{3 / 2}^{n} & =\theta_{3 / 2}^{n}-\theta_{3 / 2}^{0} \gamma_{0} \gamma^{n}, \\
\tilde{\Phi}_{3 / 2}^{0} & =\Phi_{3 / 2}^{0}+\left(-\partial_{m}+\frac{i}{2} M_{3 / 2} \gamma_{m}\right) \tilde{\theta}_{3 / 2}^{m}, \\
\tilde{\theta}^{n \ddagger} & =\theta_{3 / 2}^{n}+\gamma^{n} \gamma^{0} \theta_{3 / 2}^{0}{ }^{\ddagger}, \\
\tilde{\Phi}_{3 / 2}^{0 \ddagger} & =\Phi_{3 / 2}^{0}+\tilde{\theta}_{3 / 2}^{m \ddagger}\left(-\overleftarrow{\partial}_{m}+\frac{i}{2} M_{3 / 2} \gamma_{m}\right), \\
\tilde{\Phi}_{2}^{n} & =\Phi_{2}^{n}-2 \partial^{n} \theta_{2}^{00},
\end{aligned}
$$

\footnotetext{
${ }^{9}$ In this case also $\partial_{n} \theta_{3 / 2}^{n}$ and $\partial_{n} \theta_{3 / 2}^{n}{ }^{\ddagger}$ become first class.

${ }^{10}$ Actually all constraints become first class.
} 


$$
\begin{aligned}
\tilde{\Phi}_{2}^{0} & =\Phi_{2}^{0}+2 \partial_{n} \theta_{2}^{n 0}, \\
\tilde{\Phi}_{2}^{(1)} & =\Phi_{2}^{(1)}-\left(2 \partial^{k} \partial_{k}+3 M_{2}^{2}\right) \theta_{2}^{00}-2 \partial_{n} \tilde{\Phi}_{2}^{n} .
\end{aligned}
$$

The remaining non-vanishing Pb's are

$$
\begin{aligned}
\left\{\theta_{1}^{0}(x), \Phi_{1}^{0}(y)\right\}_{P} & =-M_{1}^{2} \delta^{3}(x-y), \\
\left\{\tilde{\theta}_{3 / 2}^{n}(x), \tilde{\theta}_{3 / 2}^{m \ddagger}(y)\right\}_{P} & =-i \sigma^{m n} \delta^{3}(x-y), \\
\left\{\tilde{\Phi}_{3 / 2}^{0}(x), \tilde{\Phi}_{3 / 2}^{0 \ddagger}(y)\right\}_{P} & =-\frac{3 i}{2} M_{3 / 2}^{2} \delta^{3}(x-y), \\
\left\{\theta_{3 / 2}^{0}(x), \Phi_{3 / 2}^{(1)}(y)\right\}_{P} & =\gamma^{0} \delta^{3}(x-y), \\
\left\{\theta_{2}^{00}(x), \Phi_{2}^{(2)}(y)\right\}_{P} & =-\delta^{3}(x-y), \\
\left\{\tilde{\Phi}_{2}^{0}(x), \tilde{\Phi}_{2}^{(1)}(y)\right\}_{P} & =3 M_{2}^{4} \delta^{3}(x-y), \\
\left\{\theta_{2}^{0 n}(x), \tilde{\Phi}_{2}^{m}(y)\right\}_{P} & =M_{2}^{2} g^{n m} \delta^{3}(x-y) .
\end{aligned}
$$

In a proper (quantum) theory we want the constraint to vanish. Although, here, they vanish in the weak sense there still exist non-vanishing $\mathrm{Pb}$ relations among them. This means in a quantum theory that ETC and ETAC relations exist among the constraints. We, therefore, introduce the new $\mathrm{Pb}$ à la Dirac 7]: The Dirac bracket (Db), such that the Db among the constraints vanishes

$$
\begin{aligned}
\{E(x), F(y)\}_{D}= & \{E(x), F(y)\}_{P}-\int d^{3} z_{z} d^{3} z_{2}\left\{E(x), \theta_{a}\left(z_{1}\right)\right\}_{P} \\
& \times C_{a b}\left(z_{1}-z_{2}\right)\left\{\theta_{b}\left(z_{2}\right), F(y)\right\}_{P},
\end{aligned}
$$

where the inverse functions $C_{a b}\left(z_{1}-z_{2}\right)$ are defined as follows

$$
\int d^{3} z\left\{\theta_{a}(x), \theta_{c}(z)\right\}_{P} C_{c b}(z-y)=\delta_{a b} \delta^{3}(x-y),
$$

and can be deduced from (18).

The ETC and ETAC relations are obtained by multiplying the Db by a factor of $i$ 11. What we get is

$$
\begin{aligned}
& {\left[A^{0}(x), A^{n}(y)\right]_{0}=\frac{i \partial^{n}}{M_{1}^{2}} \delta^{3}(x-y), } \\
& {\left[\dot{A}^{0}(x), A^{0}(y)\right]_{0}=-\frac{i}{M_{1}^{2}} \partial^{n} \partial_{n} \delta^{3}(x-y), } \\
& {\left[\dot{A}^{n}(x), A^{m}(y)\right]_{0}=i\left(g^{n m}+\frac{\partial^{n} \partial^{m}}{M_{1}^{2}}\right) \delta^{3}(x-y), } \\
\left\{\psi^{0}(x), \psi^{0 \dagger}(y)\right\}_{0}= & -\frac{2}{3 M_{3 / 2}^{2}} \nabla^{2} \delta^{3}(x-y), \\
\left\{\psi^{0}(x), \psi^{m \dagger}(y)\right\}_{0}= & \frac{1}{M_{3 / 2}}\left[\frac{2}{3 M_{3 / 2}}\left(i \gamma^{k} \partial_{k}\right) \gamma^{0} i \partial^{m}+\frac{1}{3}\left(i \gamma^{k} \partial_{k}\right) \gamma^{0} \gamma^{m}+\gamma^{0} i \partial^{m}\right] \delta^{3}(x-y), \\
\left\{\psi^{n}(x), \psi^{0 \dagger}(y)\right\}_{0}= & \frac{1}{M_{3 / 2}}\left[\frac{2}{3 M_{3 / 2}}\left(i \gamma^{k} \partial_{k}\right) i \partial^{n} \gamma^{0}+\frac{1}{3} \gamma^{n} \gamma^{0}\left(i \gamma^{k} \partial_{k}\right)+i \partial^{n} \gamma^{0}\right] \delta^{3}(x-y), \\
\left\{\psi^{n}(x), \psi^{m \dagger}(y)\right\}_{0}= & -\left[g^{n m}-\frac{1}{3} \gamma^{n} \gamma^{m}+\frac{2}{3 M_{3 / 2}^{2}} \partial^{n} \partial^{m}+\frac{1}{3 M_{3 / 2}}\left(\gamma^{n} i \partial^{m}-i \partial^{n} \gamma^{m}\right)\right] \delta^{3}(x-y),
\end{aligned}
$$

\footnotetext{
${ }^{11}$ Of course, this is not the only step to be made when passing to a quantum theory. Also the fields should be regarded as state operators, etc.
} 


$$
\begin{aligned}
{\left[h^{00}(x), h^{0 l}(y)\right]_{0} } & =\frac{4 i}{3 M_{2}^{4}} \partial^{j} \partial_{j} \partial^{l} \delta^{3}(x-y) \\
{\left[h^{0 m}(x), h^{k l}(y)\right]_{0}=} & \frac{-i}{M_{2}^{2}}\left[\frac{4}{3 M^{2}} \partial^{m} \partial^{k} \partial^{l}-\frac{2}{3} \partial^{m} g^{k l}+\partial^{k} g^{m l}+\partial^{l} g^{m k}\right] \delta^{3}(x-y) \\
{\left[\dot{h}^{00}(x), h^{00}(y)\right]_{0}=} & -\frac{4 i}{3 M_{2}^{4}} \partial^{i} \partial_{i} \partial^{j} \partial_{j} \delta^{3}(x-y) \\
{\left[\dot{h}^{0 m}(x), h^{0 l}(y)\right]_{0}=} & \frac{i}{M_{2}^{2}}\left[\frac{4}{3 M_{2}^{2}} \partial^{m} \partial^{l} \partial^{j} \partial_{j}+\frac{1}{3} \partial^{m} \partial^{l}+\partial^{j} \partial_{j} g^{m l}\right] \delta^{3}(x-y) \\
{\left[\dot{h}^{00}(x), h^{k l}(y)\right]_{0}=} & \frac{i}{M_{2}^{2}}\left[\frac{4}{3 M_{2}^{2}} \partial^{k} \partial^{l} \partial^{j} \partial_{j}+2 \partial^{k} \partial^{l}-\frac{2}{3} \partial^{j} \partial_{j} g^{k l}\right] \delta^{3}(x-y) \\
{\left[\dot{h}^{n m}(x), h^{k l}(y)\right]_{0}=} & i\left[-g^{n k} g^{m l}-g^{n l} g^{m k}+\frac{2}{3} g^{n m} g^{k l}\right. \\
& -\frac{1}{M_{2}^{2}}\left(\partial^{n} \partial^{k} g^{m l}+\partial^{m} \partial^{k} g^{n l}+\partial^{n} \partial^{l} g^{m k}+\partial^{m} \partial^{l} g^{n k}\right) \\
& \left.\quad+\frac{2}{3 M_{2}^{2}}\left(\partial^{n} \partial^{m} g^{k l}+g^{n m} \partial^{k} \partial^{l}\right)-\frac{4}{3 M_{2}^{2}} \partial^{n} \partial^{m} \partial^{k} \partial^{l}\right] \delta^{3}(x-y)
\end{aligned}
$$

This concludes the quantization of free, massive higher spin $(j=1,3 / 2,2)$ fields. As a final remark we notice that the ET(A)C relations in (21) amongst the various components of the spin-3/2, spin-2 field and their velocities are independent of the choice of the parameter $A$ in (1).

\subsection{Propagators}

Having quantized the free fields in the previous subsection (section 2.2) we now want to obtain the propagators. In order to do so we need to calculate the commutation relations for non-equal times, which is done using the following identities as solutions to the field equations (first column of (4))

$$
\begin{aligned}
A^{\mu}(x) & =\int d^{3} z\left[\partial_{0}^{z} \Delta\left(x-z ; M_{1}^{2}\right) A^{\mu}(z)-\Delta\left(x-z ; M_{1}^{2}\right) \partial_{0}^{z} A^{\mu}(z)\right], \\
\psi^{\mu}(x) & =i \int d^{3} z\left(i \partial_{x}+M_{3 / 2}\right) \gamma_{0} \Delta\left(x-z ; M_{3 / 2}^{2}\right) \psi^{\mu}(z), \\
h^{\mu \nu}(x) & =\int d^{3} z\left[\partial_{0}^{z} \Delta\left(x-z ; M_{2}^{2}\right) h^{\mu \nu}(z)-\Delta\left(x-z ; M_{2}^{2}\right) \partial_{0}^{z} h^{\mu \nu}(z)\right] .
\end{aligned}
$$

Using these equations (22) and the ETC and ETAC relations we obtained before (21) we calculate the commutation relations for unequal times

$$
\begin{aligned}
{\left[A^{\mu}(x), A^{\nu}(y)\right]=} & -i\left(g^{\mu \nu}+\frac{\partial^{\mu} \partial^{\nu}}{M_{1}^{2}}\right) \Delta\left(x-y ; M_{1}^{2}\right)=P_{1}^{\mu \nu}(\partial) i \Delta\left(x-y ; M_{1}^{2}\right), \\
\left\{\psi^{\mu}(x), \bar{\psi}^{\nu}(y)\right\}= & -i\left(i \not \partial+M_{3 / 2}\right)\left[g^{\mu \nu}-\frac{1}{3} \gamma^{\mu} \gamma^{\nu}+\frac{2 \partial^{\mu} \partial^{\nu}}{3 M_{3 / 2}^{2}}-\frac{1}{3 M_{3 / 2}}\left(\gamma^{\mu} i \partial^{\nu}-\gamma^{\nu} i \partial^{\mu}\right)\right] \\
& \quad \times \Delta\left(x-y ; M_{3 / 2}^{2}\right)=\left(i \not \partial+M_{3 / 2}\right) P_{3 / 2}^{\mu \nu}(\partial) i \Delta\left(x-y ; M_{3 / 2}^{2}\right), \\
{\left[h^{\mu \nu}(x), h^{\alpha \beta}(y)\right]=\quad } & i\left[g^{\mu \alpha} g^{\nu \beta}+g^{\mu \beta} g^{\nu \alpha}-\frac{2}{3} g^{\mu \nu} g^{\alpha \beta}\right. \\
& +\frac{1}{M_{2}^{2}}\left(\partial^{\mu} \partial^{\alpha} g^{\nu \beta}+\partial^{\nu} \partial^{\alpha} g^{\mu \beta}+\partial^{\mu} \partial^{\beta} g^{\nu \alpha}+\partial^{\nu} \partial^{\beta} g^{\mu \alpha}\right) \\
& \left.\quad-\frac{2}{3 M_{2}^{2}}\left(\partial^{\mu} \partial^{\nu} g^{\alpha \beta}+g^{\mu \nu} \partial^{\alpha} \partial^{\beta}\right)+\frac{4}{3 M_{2}^{2}} \partial^{\mu} \partial^{\nu} \partial^{\alpha} \partial^{\beta}\right] \Delta\left(x-y ; M_{2}^{2}\right) \\
= & 2 P_{2}^{\mu \nu \alpha \beta}(\partial) i \Delta\left(x-y ; M_{2}^{2}\right),
\end{aligned}
$$

where the $P_{j}(\partial), j=1,3 / 2,2$ are the (on mass shell) spin projection operators. The factor 2 in the last line of (23) can be transformed away by redefining the spin-2 field. Equation (23) yields for the 
propagators

$$
\begin{aligned}
& D_{F}^{\mu \nu}(x-y)=-i<0\left|T\left[A^{\mu}(x) A^{\nu}(y)\right]\right| 0> \\
& =-i \theta\left(x^{0}-y^{0}\right) P_{1}^{\mu \nu}(\partial) \Delta^{(+)}\left(x-y ; M_{1}^{2}\right)-i \theta\left(y^{0}-x^{0}\right) P_{1}^{\mu \nu}(\partial) \Delta^{(-)}\left(x-y ; M_{1}^{2}\right) \\
& =P_{1}^{\mu \nu}(\partial) \Delta_{F}\left(x-y ; M_{1}^{2}\right)-i \delta_{0}^{\mu} \delta_{0}^{\nu} \delta^{4}(x-y) \text {. } \\
& S_{F}^{\mu \nu}(x-y)=-i<0\left|T\left(\psi^{\mu}(x) \bar{\psi}^{\nu}(y)\right)\right| 0> \\
& =-i \theta\left(x^{0}-y^{0}\right)\left(i \not \partial+M_{3 / 2}\right) P_{3 / 2}^{\mu \nu}(\partial) \Delta^{(+)}\left(x-y ; M_{3 / 2}^{2}\right) \\
& -i \theta\left(y^{0}-x^{0}\right)\left(i \not \partial+M_{3 / 2}\right) P_{3 / 2}^{\mu \nu}(\partial) \Delta^{(-)}\left(x-y ; M_{3 / 2}^{2}\right) \\
& =\left(i \not \partial+M_{3 / 2}\right) P_{3 / 2}^{\mu \nu}(\partial) \Delta_{F}\left(x-y ; M_{3 / 2}^{2}\right) \\
& -\gamma_{0}\left[\frac{2}{3 M_{3 / 2}^{2}}\left(\delta_{0}^{\mu} \delta_{m}^{\nu}+\delta_{0}^{\nu} \delta_{m}^{\mu}\right) i \partial^{m}+\frac{1}{3 M_{3 / 2}}\left(\delta_{m}^{\mu} \delta_{0}^{\nu}-\delta_{m}^{\nu} \delta_{0}^{\mu}\right) \gamma^{m}\right] \delta^{4}(x-y) \\
& -\frac{2}{3 M_{3 / 2}^{2}}\left(i \not \partial+M_{3 / 2}\right) \delta_{0}^{\mu} \delta_{0}^{\nu} \delta^{4}(x-y) \text {. } \\
& D_{F}^{\mu \nu \alpha \beta}(x-y)=-i<0\left|T\left[h^{\mu \nu}(x) h^{\alpha \beta}(y)\right]\right| 0> \\
& =-i \theta\left(x^{0}-y^{0}\right) 2 P_{2}^{\mu \nu \alpha \beta}(\partial) \Delta^{(+)}\left(x-y ; M_{2}^{2}\right)-i \theta\left(y^{0}-x^{0}\right) 2 P_{2}^{\mu \nu \alpha \beta}(\partial) \Delta^{(-)}\left(x-y ; M_{2}^{2}\right) \\
& =2 P_{2}^{\mu \nu \alpha \beta}(\partial) \Delta_{F}\left(x-y ; M_{2}^{2}\right) \\
& +\frac{1}{M_{2}^{2}}\left[\delta_{0}^{\mu} \delta_{0}^{\alpha} g^{\nu \beta}+\delta_{0}^{\nu} \delta_{0}^{\alpha} g^{\mu \beta}+\delta_{0}^{\mu} \delta_{0}^{\beta} g^{\nu \alpha}+\delta_{0}^{\nu} \delta_{0}^{\beta} g^{\mu \alpha}\right. \\
& -\frac{2}{3}\left(\delta_{0}^{\mu} \delta_{0}^{\nu} g^{\alpha \beta}+g^{\mu \nu} \delta_{0}^{\alpha} \delta_{0}^{\beta}\right)+\frac{4}{3}\left(\delta_{0}^{\mu} \delta_{0}^{\nu} \delta_{0}^{\alpha} \delta_{0}^{\beta}\left(\partial^{0} \partial_{0}-\partial^{k} \partial_{k}-M_{2}^{2}\right)\right. \\
& +\delta_{0}^{\mu} \delta_{0}^{\nu} \delta_{0}^{\alpha} \delta_{b}^{\beta} \partial^{0} \partial^{b}+\delta_{0}^{\mu} \delta_{0}^{\nu} \delta_{a}^{\alpha} \delta_{0}^{\beta} \partial^{0} \partial^{a}+\delta_{0}^{\mu} \delta_{n}^{\nu} \delta_{0}^{\alpha} \delta_{0}^{\beta} \partial^{0} \partial^{n}+\delta_{m}^{\mu} \delta_{0}^{\nu} \delta_{0}^{\alpha} \delta_{0}^{\beta} \partial^{0} \partial^{m} \\
& +\delta_{0}^{\mu} \delta_{0}^{\nu} \delta_{a}^{\alpha} \delta_{b}^{\beta} \partial^{a} \partial^{b}+\delta_{0}^{\mu} \delta_{n}^{\nu} \delta_{0}^{\alpha} \delta_{b}^{\beta} \partial^{n} \partial^{b}+\delta_{m}^{\mu} \delta_{0}^{\nu} \delta_{0}^{\alpha} \delta_{b}^{\beta} \partial^{m} \partial^{b}+\delta_{0}^{\mu} \delta_{n}^{\nu} \delta_{a}^{\alpha} \delta_{0}^{\beta} \partial^{n} \partial^{a} \\
& \left.\left.+\delta_{m}^{\mu} \delta_{0}^{\nu} \delta_{a}^{\alpha} \delta_{0}^{\beta} \partial^{m} \partial^{a}+\delta_{m}^{\mu} \delta_{n}^{\nu} \delta_{0}^{\alpha} \delta_{0}^{\beta} \partial^{m} \partial^{n}\right)\right] \delta^{4}(x-y) .
\end{aligned}
$$

The use of $\Delta^{(+)}(x-y)$ and $\Delta^{(-)}(x-y)$ is similar to what is written in [43] in case of scalar fields

$$
\begin{aligned}
& <0|\phi(x) \phi(y)| 0>=\Delta^{(+)}(x-y), \\
& <0|\phi(y) \phi(x)| 0>=\Delta^{(-)}(x-y) .
\end{aligned}
$$

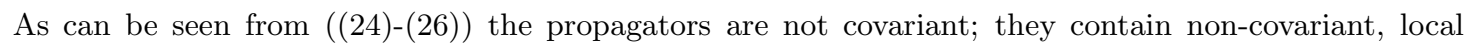
terms, as is mentioned in for instance [15].

\section{$3 \quad$ Auxiliary Fields}

The goal of this section is to come to covariant propagators. The way we do this is to introduce auxiliary fields. Since we also allow for mass terms we have extra parameters which can be seen as gauge parameters. We discuss certain choices of these parameters. Also we discuss the massless limits of the propagators in section 3.4 and give momentum representations of the fields in section 3.5 Apart from that, the organization of this section is exactly the same as the previous one (section 21).

\subsection{Equations of Motion}

As a starting point we take the Lagrangians (1a), (2a) and (2b). To these Lagrangians we add auxiliary fields coupled to the gauge conditions of the massless theory, as discussed in the text below (4). We also 
allow for mass terms of these auxiliary fields, which introduces parameters to be seen as gauge parameters

$$
\begin{aligned}
\mathcal{L}_{B} & =\mathcal{L}_{1}+M_{1} B \partial^{\mu} A_{\mu}+\frac{1}{2} a M_{1}^{2} B^{2} \\
\mathcal{L}_{\chi} & =\mathcal{L}_{3 / 2}+M_{3 / 2} \bar{\chi} \gamma^{\mu} \psi_{\mu}+M_{3 / 2} \bar{\psi}_{\mu} \gamma^{\mu} \chi+b M_{3 / 2} \bar{\chi} \chi \\
\mathcal{L}_{\eta \epsilon} & =\mathcal{L}_{2}+M_{2} \partial_{\mu} h^{\mu \nu} \eta_{\nu}+M_{2}^{2} h_{\mu}^{\mu} \epsilon+\frac{1}{2} c M_{2}^{2} \eta^{\mu} \eta_{\mu} .
\end{aligned}
$$

In (28c) we did not allow for a mass term for the $\epsilon$ field. We will come back to this point below.

These Lagrangians (28a)-(28c) ) lead to the following EoM's.

$$
\begin{aligned}
\left(\square+M_{1}^{2}\right) A^{\mu} & =(1-a) M_{1} \partial^{\mu} B, \\
\left(\square+M_{B}^{2}\right)\left(\square+M_{1}^{2}\right) A^{\mu} & =0, \\
\left(\square+M_{B}^{2}\right) B & =0,
\end{aligned}
$$

where $M_{B}^{2}=a M_{1}^{2}$. Furthermore we have the constraint relation $\partial^{\mu} A_{\mu}=-a M_{1} B$.

$$
\begin{aligned}
\left(i \not \partial-M_{3 / 2}\right) \psi_{\mu} & =-\frac{b+2}{2} M_{3 / 2} \gamma_{\mu} \chi-b i \partial_{\mu} \chi \\
\left(i \not \partial+M_{\chi}\right)\left(i \not \partial-M_{3 / 2}\right) \psi_{\mu} & =-\left(3 b^{2}+5 b+2\right) M_{3 / 2} i \partial_{\mu} \chi \\
\left(\square+M_{\chi}^{2}\right)\left(i \not \partial-M_{3 / 2}\right) \psi_{\mu} & =0 \\
\left(i \not \partial-M_{\chi}\right) \chi & =0
\end{aligned}
$$

where $M_{\chi}=(3 b / 2+2) M_{3 / 2}$. The auxiliary field is related to the original spin- $3 / 2$ field via the equations $\gamma \cdot \psi=-b \chi$ and $i \partial \cdot \psi=-\frac{1}{2}(1+b)(3 b+4) M_{3 / 2} \chi$.

$$
\begin{aligned}
\left(\square+M_{2}^{2}\right) h^{\mu \nu} & =-(1+c) M_{2}\left(\partial^{\mu} \eta^{\nu}+\partial^{\nu} \eta^{\mu}\right)+\frac{2(1+c)}{1-c} M_{2}^{2} g^{\mu \nu} \epsilon, \\
\left(\square+M_{\eta}^{2}\right)\left(\square+M_{2}^{2}\right) h^{\mu \nu} & =\frac{2(1+c)^{2}}{1-c} M_{2}^{2}\left(2 \partial^{\mu} \partial^{\nu}-\frac{c}{3+c} M_{2}^{2} g^{\mu \nu}\right) \epsilon, \\
\left(\square+M_{\epsilon}^{2}\right)\left(\square+M_{\eta}^{2}\right)\left(\square+M_{2}^{2}\right) h^{\mu \nu} & =0, \\
\left(\square+M_{\eta}^{2}\right) \eta^{\mu} & =-\frac{2(1+c)}{1-c} M_{2} \partial^{\mu} \epsilon, \\
\left(\square+M_{\epsilon}^{2}\right)\left(\square+M_{\eta}^{2}\right) \eta^{\mu} & =0, \\
\left(\square+M_{\epsilon}^{2}\right) \epsilon & =0,
\end{aligned}
$$

where $M_{\eta}^{2}=-c M_{2}^{2}$ and $M_{\epsilon}^{2}=-\frac{2 c}{3+c} M_{2}^{2}$. The constraint relations are $h_{\mu}^{\mu}=0, \partial_{\mu} h^{\mu \nu}=-c M_{2} \eta^{\nu}$ and $\partial \cdot \eta=\frac{4 M_{2}}{1-c} \epsilon$

From the last line of (31) we see that the $\epsilon$-field is a free Klein-Gordon field. This equation comes about quite naturally from the Euler-Lagrange equations. This would not be so if we allowed for a mass term of this $\epsilon$-field in the Lagrangian (28c). Then it must be imposed that $\epsilon$ is a free Klein-Gordon field which makes the calculations unnatural and unnecessary difficult. 


\subsection{Quantization}

As mentioned before the quantization procedure runs exactly the same as in the previous section (section 2.2). We, therefore, determine the canonical momenta to be

$$
\begin{array}{ll}
\pi_{1}^{0}=M_{1} B, & \pi_{B}=0, \\
\pi_{1}^{n}=-\dot{A}^{n}+\partial^{n} A^{0}, & \\
& \\
\pi_{3 / 2}^{0}=0, & \pi_{3 / 2}^{0} \ddagger=0, \\
\pi_{3 / 2}^{n}=\frac{i}{2} \psi_{k}^{\dagger} \sigma^{k n}, & \pi_{3 / 2}^{n}=\frac{i}{2} \sigma^{n k} \psi_{k}, \\
\pi_{\chi}=0, & \pi_{\chi}^{\ddagger}=0, \\
\pi_{2}^{00}=-\frac{1}{2} \partial_{n} h^{n 0}+M_{2} \eta^{0}, & \pi_{\eta}^{0}=0, \\
\pi_{2}^{0 m}=-\partial_{n} h^{n m}+\frac{1}{2} \partial^{m} h^{00}+\frac{1}{2} \partial^{m} h_{n}^{n}+M_{2} \eta^{m}, & \pi_{\eta}^{m}=0, \\
\pi_{2}^{n m}=\frac{1}{2} \dot{h}^{n m}-\frac{1}{2} g^{n m} \dot{h}_{k}^{k}+\frac{1}{2} g^{n m} \partial_{k} h^{k 0}, & \pi_{\epsilon}=0, \\
&
\end{array}
$$

from which we deduce the velocities

$$
\begin{aligned}
\dot{A}^{n} & =-\pi_{1}^{n}+\partial^{n} A^{0}, \\
\dot{h}^{n m} & =2 \pi_{2}^{n m}-g^{n m} \pi_{2 k}^{k}+\frac{1}{2} g^{n m} \partial_{k} h^{k 0}, \\
\dot{h}_{k}^{k} & =-\pi_{2 k}^{k}+\frac{3}{2} \partial_{k} h^{k 0} .
\end{aligned}
$$

These velocities are the same as in the previous section (see (17)). The primary constraints are

$$
\begin{aligned}
& \theta_{1}^{0}=\pi_{1}^{0}-M_{1} B, \quad \theta_{B}=\pi_{B}, \\
& \theta_{3 / 2}^{0}=\pi_{3 / 2}^{0}, \quad \theta_{3 / 2}^{0}{ }^{\ddagger}=\pi_{3 / 2}^{0}{ }^{\ddagger}, \\
& \theta_{3 / 2}^{n}=\pi_{3 / 2}^{n}-\frac{i}{2} \psi_{k}^{\dagger} \sigma^{k n}, \quad \theta_{3 / 2}^{n}=\pi_{3 / 2}^{n}{ }^{\ddagger}-\frac{i}{2} \sigma^{n k} \psi_{k}, \\
& \theta_{\chi}=\pi_{\chi}, \quad \theta_{\chi}^{\ddagger}=\pi_{\chi}^{\ddagger}, \\
& \theta_{2}^{00}=\pi_{2}^{00}+\frac{1}{2} \partial_{n} h^{n 0}-M_{2} \eta^{0}, \quad \theta_{\eta}^{0}=\pi_{\eta}^{0},
\end{aligned}
$$

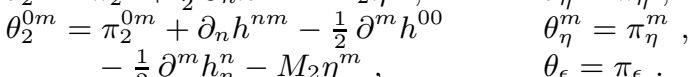

Having determined the canonical momenta, the velocities and the primary constraints we determine the (strong) Hamiltonians to be

$$
\begin{aligned}
\mathcal{H}_{B, S}= & -\frac{1}{2} \pi_{1}^{n} \pi_{1, n}+\pi_{1}^{n} \partial_{n} A_{0}+\frac{1}{2} \partial_{m} A_{n} \partial^{m} A^{n}-\frac{1}{2} \partial_{m} A_{n} \partial^{n} A^{m}-\frac{1}{2} M_{1}^{2} A^{0} A_{0} \\
& -\frac{1}{2} M_{1}^{2} A^{n} A_{n}-M_{1} B \partial^{m} A_{m}-\frac{1}{2} a M_{1}^{2} B^{2}+\lambda_{1,0} \theta_{1}^{0}+\lambda_{B} \theta_{B}, \\
\mathcal{H}_{\chi, S}= & \frac{1}{2} \epsilon^{\mu \nu \rho k} \bar{\psi}_{\mu} \gamma_{5} \gamma_{\rho}\left(\partial_{k} \psi_{\nu}\right)-\frac{1}{2} \epsilon^{\mu \nu \rho k}\left(\partial_{k} \bar{\psi}_{\mu}\right) \gamma_{5} \gamma_{\rho} \psi_{\nu}+M_{3 / 2} \bar{\psi}_{\mu} \sigma^{\mu \nu} \psi_{\nu} \\
& -M_{3 / 2} \bar{\chi} \gamma^{\mu} \psi_{\mu}-M_{3 / 2} \bar{\psi}_{\mu} \gamma^{\mu} \chi-b M_{3 / 2} \bar{\chi} \chi+\lambda_{3 / 2,0} \theta_{3 / 2}^{0}+\lambda_{3 / 2, n} \theta_{3 / 2}^{n} \\
& +\lambda_{3 / 2,0}^{\ddagger} \theta_{3 / 2}^{0}+\lambda_{3 / 2, n}^{\ddagger} \theta_{3 / 2}^{n}+\lambda_{\chi} \theta_{\chi}+\lambda_{\chi}^{\ddagger} \theta_{\chi}^{\ddagger}, \\
\mathcal{H}_{\eta \epsilon, S} & \pi_{2}^{n m} \pi_{2, n m}-\frac{1}{2} \pi_{2}^{n} \pi_{2}^{m}+\frac{1}{2} \pi_{2}^{n} \partial^{m} h_{m 0}-\frac{1}{2} \partial^{k} h^{n 0} \partial_{k} h_{n 0}-\frac{1}{4} \partial^{k} h^{n m} \partial_{k} h_{n m} \\
& +\frac{1}{8} \partial_{n} h^{n 0} \partial^{m} h_{m 0}+\frac{1}{2} \partial_{n} h^{n m} \partial^{k} h_{k m}+\frac{1}{2} \partial_{m} h^{00} \partial^{m} h_{n}^{n}+\frac{1}{4} \partial_{m} h_{n}^{n} \partial^{m} h_{k}^{k} \\
& -\frac{1}{2} \partial_{n} h^{n m} \partial_{m} h_{00}-\frac{1}{2} \partial_{n} h^{n m} \partial_{m} h_{k}^{k}+\frac{1}{2} M_{2}^{2} h^{n 0} h_{n 0}+\frac{1}{4} M_{2}^{2} h^{n m} h_{n m}
\end{aligned}
$$




$$
\begin{aligned}
& -\frac{1}{2} M_{2}^{2} h^{00} h_{m}^{m}-\frac{1}{4} M_{2}^{2} h_{n}^{n} h_{m}^{m}-\frac{1}{2} c M_{2}^{2} \eta^{\mu} \eta_{\mu}-M_{2} \partial_{n} h^{n 0} \eta_{0}-M_{2} \partial_{n} h^{n m} \eta_{m} \\
& -M_{2}^{2} h_{0}^{0} \epsilon-M_{2}^{2} h_{k}^{k} \epsilon+\lambda_{2,00} \theta_{2}^{00}+\lambda_{2,0 m} \theta_{2}^{0 m}+\lambda_{0, \eta} \theta_{\eta}^{0}+\lambda_{m, \eta} \theta_{\eta}^{m}+\lambda_{\epsilon} \theta_{\epsilon} .
\end{aligned}
$$

With this Hamiltonians (35) and with the definition of the $\mathrm{Pb}$ in (10) we impose the time-derivatives of the constraints (34) to be zero

$$
\begin{aligned}
& \left\{\theta_{1}^{0}(x), H_{B, S}\right\}_{P}=\partial_{n} \pi_{1}^{n}+M_{1}^{2} A^{0}-M_{1} \lambda_{B}=0, \\
& \left\{\theta_{B}(x), H_{B, S}\right\}_{P}=M_{1} \partial^{m} A_{m}+a M_{1}^{2} B+M_{1} \lambda_{1,0}=0,
\end{aligned}
$$

$$
\begin{aligned}
\left\{\theta_{3 / 2}^{0}(x), H_{\chi, S}\right\}_{P} & =\epsilon^{\mu 0 \rho k}\left(\partial_{k} \bar{\psi}_{\mu}\right) \gamma_{5} \gamma_{\rho}-M_{3 / 2} \bar{\psi}_{\mu} \sigma^{\mu 0}+M_{3 / 2} \bar{\chi} \gamma^{0}=0 \equiv-\Phi_{3 / 2}^{0}{ }^{\ddagger}(x), \\
\left\{\theta_{3 / 2}^{0}{ }^{\ddagger}(x), H_{\chi, S}\right\}_{P} & =-\epsilon^{\mu 0 \rho k} \gamma^{0} \gamma_{5} \gamma_{\rho}\left(\partial_{k} \psi_{\mu}\right)+M_{3 / 2} \gamma^{0} \sigma^{0 \mu} \psi_{\mu}-M_{3 / 2} \chi=0 \\
\left\{\theta_{3 / 2}^{n}(x), H_{\chi, S}\right\}_{P} & \equiv-\Phi_{3 / 2}^{0}(x), \\
\left\{\theta_{3 / 2}^{n}{ }^{\mu n \rho k}(x), H_{\chi, S}\right\}_{P} & \left.=-\epsilon^{\mu n \rho k} \bar{\psi}_{\mu}\right) \gamma_{5} \gamma_{\rho}-M_{3 / 2} \gamma_{\rho}\left(\partial_{\mu} \sigma^{\mu n}+M \bar{\chi} \gamma^{n}+i \lambda_{3 / 2, k}^{\ddagger} \sigma^{k n}=0,\right. \\
\left\{\theta_{\chi}(x), H_{\chi, S}\right\}_{P} & =M_{3 / 2} \bar{\psi} \cdot \gamma+b \sigma_{3 / 2} \bar{\chi}=0 \equiv-M_{3 / 2} \Phi_{\chi}^{\ddagger} \gamma^{0}, M \gamma^{0} \gamma^{n} \chi+i \sigma^{n k} \lambda_{3 / 2, k}=0, \\
\left\{\theta_{\chi}^{\ddagger}(x), H_{\chi, S}\right\}_{P} & =-M_{3 / 2} \gamma^{0} \gamma \cdot \psi-b M_{3 / 2} \gamma^{0} \chi=0 \equiv-M_{3 / 2} \gamma^{0} \Phi_{\chi},
\end{aligned}
$$

$$
\begin{aligned}
\left\{\theta_{2}^{00}(x), H_{\eta \epsilon, S}\right\}_{P} & =-M_{2} \lambda_{\eta}^{0}+\frac{1}{2}\left(\partial^{k} \partial_{k}+M_{2}^{2}\right) h_{m}^{m}-\frac{1}{2} \partial_{n} \partial_{m} h^{n m}+M_{2}^{2} \epsilon=0, \\
\left\{\theta_{2}^{0 m}(x), H_{\eta \epsilon, S}\right\}_{P} & =2 \partial_{k} \pi_{2}^{k m}-\left(\partial^{k} \partial_{k}+M_{2}^{2}\right) h^{0 m}-M_{2} \partial^{m} \eta^{0}-M_{2} \lambda_{\eta}^{m}=0, \\
\left\{\theta_{\eta}^{0}(x), H_{\eta \epsilon, S}\right\}_{P} & =\partial_{n} h^{n 0}+\lambda_{2}^{00}+c M_{2} \eta^{0}=0, \\
\left\{\theta_{\eta}^{m}(x), H_{\eta \epsilon, S}\right\}_{P} & =\partial_{n} h^{n m}+\lambda_{2}^{0 m}+c M_{2} \eta^{m}=0, \\
\left\{\theta_{\epsilon}(x), H_{\eta \epsilon, S}\right\}_{P} & =M_{2}^{2}\left[h_{0}^{0}+h_{n}^{n}\right]=0 \equiv M_{2}^{2} \Phi_{\eta},
\end{aligned}
$$

Equations (36a), (36b), (37c), (37d) and (38a)-38d determine the Lagrange multipliers $\lambda_{B}, \lambda_{1,0}, \lambda_{3 / 2, k}^{\ddagger}$, $\lambda_{3 / 2, k}, \lambda_{\eta}^{0}, \lambda_{\eta}^{m}, \lambda_{2}^{00}, \lambda_{2}^{0 m}$, respectively. All other equations in (36), (37) and (38) yield new (secondary) constraints. Imposing their time derivatives to be zero, yields

$$
\begin{aligned}
\left\{\Phi_{3 / 2}^{0}(x), H_{\chi, S}\right\}_{P} & =\sigma^{n k} i \partial_{n} \lambda_{k}+M_{3 / 2} \gamma^{k} \lambda_{3 / 2, k}-M_{3 / 2} \lambda_{\chi}=0, \\
\left\{\Phi_{3 / 2}^{0}{ }^{\ddagger}(x), H_{\chi, S}\right\}_{P} & =i \partial_{n} \lambda_{3 / 2, k}^{\ddagger} \sigma^{k n}+M_{3 / 2} \lambda_{3 / 2, k}^{\ddagger} \gamma^{k}+M_{3 / 2} \lambda_{\chi}^{\ddagger}=0, \\
\left\{\Phi_{\chi}(x), H_{\chi, S}\right\}_{P} & =-b \lambda_{\chi}-\gamma^{0} \lambda_{3 / 2,0}-\gamma^{n} \lambda_{3 / 2, n}=0, \\
\left\{\Phi_{\chi}^{\ddagger}(x), H_{\chi, S}\right\}_{P} & =b \lambda_{\chi}^{\ddagger}+\lambda_{3 / 2,0}^{\ddagger} \gamma^{0}-\lambda_{3 / 2, n}^{\ddagger} \gamma^{n}=0, \\
\left\{\Phi_{\eta}(x), H_{\eta \epsilon}\right\}_{P} & =-\pi_{2 k}^{k}+\frac{1}{2} \partial_{n} h^{n 0}-c M_{2} \eta^{0}=0=-\Phi_{2}^{(1)} .
\end{aligned}
$$

The equations in (39) determine the Lagrange multipliers $\lambda_{\chi}, \lambda_{\chi}^{\ddagger}, \lambda_{3 / 2,0}$ and $\lambda_{3 / 2,0}^{\ddagger}$. Equation (40) yields yet another (tertiary) constraint. Imposing its time derivative to be zero

$$
\begin{aligned}
\left\{\Phi_{2}^{(1)}(x), H_{\eta \epsilon}\right\}_{P}= & \partial^{k} \partial_{k} h^{00}+\frac{1}{2} \partial^{k} \partial_{k} h_{m}^{m}-\frac{1}{2} \partial_{n} \partial_{m} h^{n m}+\frac{3}{2} M_{2}^{2} h^{00}+M_{2}^{2} h_{m}^{m} \\
& -M_{2} \partial^{k} \eta_{k}-\partial_{m} \lambda_{2}^{m 0}+3 M_{2}^{2} \epsilon+c M_{2} \lambda_{\eta}^{0}=0,
\end{aligned}
$$


gives an equation for $\lambda_{\eta}^{0}$. Since we already had an equation determining $\lambda_{\eta}^{0}$ (38a) we combine both equations for consistency and use $\Phi_{\eta}$ as a weakly vanishing constraint. What we get is the last constraint

$$
\begin{aligned}
\Phi_{2}^{(2)}= & -\partial_{n} \partial_{m} h^{n m}+\left(\partial^{k} \partial_{k}+M_{2}^{2}\right) h_{m}^{m}+2 M_{2} \partial^{k} \eta_{k}-2\left(\frac{3+c}{1-c}\right) M_{2}^{2} \epsilon \\
\left\{\Phi_{2}^{(2)}(x), H_{\eta \epsilon, S}\right\}_{P}= & -2 \partial_{n} \partial_{m} \pi_{2}^{n m}-M_{2}^{2} \pi_{2 k}^{k}+\left(\partial^{k} \partial_{k}+\frac{3}{2} M_{2}^{2}\right) \partial_{n} h^{n 0}+2 M_{2} \partial_{k} \lambda_{\eta}^{k} \\
& -2\left(\frac{3+c}{1-c}\right) M_{2}^{2} \lambda_{\epsilon}=0 .
\end{aligned}
$$

As can be seen in (42) imposing the time derivative of $\Phi_{2}^{(2)}$ to be zero determines the remaining Lagrange multiplier $\lambda_{\epsilon}$.

All Lagrange multipliers are determined, which, again, means that all constraints are second class. So, every constraint has at least one non-vanishing $\mathrm{Pb}$ with another constraint. The complete set of constraints is

$$
\begin{array}{ll}
\theta_{1}^{0}=\pi_{1}^{0}-M_{1} B, & \theta_{B}=\pi_{B}, \\
\theta_{3 / 2}^{0}=\pi_{3 / 2}^{0}, & \theta_{3 / 2}^{0}{ }^{\ddagger}=\pi_{3 / 2}^{0}{ }^{\ddagger}, \\
\theta_{3 / 2}^{n}=\pi_{3 / 2}^{n}-\frac{i}{2} \psi_{k}^{\dagger} \sigma^{k n}, & \theta_{3 / 2}^{n}=\pi_{3 / 2}^{n}-\frac{i}{2} \sigma^{n k} \psi_{k}, \\
\theta_{\chi}=\pi_{\chi}, & \theta_{\chi}^{\ddagger}=\pi_{\chi}^{\ddagger}, \\
\Phi_{3 / 2}^{0}=-i \sigma^{k n} \partial_{k} \psi_{n}-M_{3 / 2}\left(\gamma^{k} \psi_{k}-\chi\right), & \Phi_{3 / 2}^{0}=-i \partial_{k} \psi_{n}^{\dagger} \sigma^{n k}-M_{3 / 2}\left(\psi_{k}^{\dagger} \gamma^{k}+\chi^{\dagger}\right), \\
\Phi_{\chi}=\gamma^{0} \psi_{0}+\gamma^{k} \psi_{k}+b \chi, & \Phi_{\chi}^{\ddagger}=-\psi_{0}^{\dagger} \gamma^{0}+\psi_{k}^{\dagger} \gamma^{k}-b \chi^{\dagger}, \\
\theta_{2}^{00}=\pi_{2}^{00}+\frac{1}{2} \partial_{n} h^{n 0}-M_{2} \eta^{0}, & \theta_{\eta}^{0}=\pi_{\eta}^{0}, \\
\theta_{2}^{0 m}=\pi_{2}^{0 m}+\partial_{n} h^{n m}-\frac{1}{2} \partial^{m} h^{00} & \theta_{\eta}^{m}=\pi_{\eta}^{m}, \\
\quad-\frac{1}{2} \partial^{m} h_{n}^{n}-M_{2} \eta^{m}, & \theta_{\epsilon}=\pi_{\epsilon}, \\
\Phi_{2}^{(2)}=-\partial_{n} \partial_{m} h^{n m}+\left(\partial^{k} \partial_{k}+M_{2}^{2}\right) h_{m}^{m}, & \Phi_{\eta}=h_{0}^{0}+h_{n}^{n}, \\
\quad+2 M_{2} \partial^{k} \eta_{k}-2\left(\frac{3+c}{1-c}\right) M_{2}^{2} \epsilon, & \Phi_{2}^{(1)}=\pi_{2 k}^{k}-\frac{1}{2} \partial_{n} h^{n 0}+c M_{2} \eta^{0} .
\end{array}
$$

Again we make linear combinations of constraints in order to reduce the number of non-vanishing Pb's

$$
\begin{aligned}
\tilde{\Phi}_{\chi} & =\Phi_{\chi}-\frac{b}{M_{3 / 2}} \Phi_{3 / 2}^{0}, \\
\tilde{\theta}_{3 / 2}^{n} & =\theta_{3 / 2}^{n}-\theta_{3 / 2}^{0} \gamma_{0}\left[(1+b) \gamma^{n}-\frac{b}{M_{3 / 2}} i \overleftarrow{\partial_{k}} \sigma^{k n}\right]+\frac{1}{M_{3 / 2}} \theta_{\chi}\left[M_{3 / 2} \gamma^{n}-i \overleftarrow{\partial_{k}} \sigma^{k n}\right], \\
\tilde{\Phi}_{\chi}^{\ddagger} & =\Phi_{\chi}^{\ddagger}-\frac{b}{M_{3 / 2}} \Phi_{3 / 2}^{0} \ddagger \\
\tilde{\theta}_{3 / 2}^{n \ddagger} & =\theta_{3 / 2}^{n}-\left[-(1+b) \gamma^{n}+\frac{b}{M_{3 / 2}} \sigma^{n k} i \partial_{k}\right] \gamma_{0} \theta_{3 / 2}^{0}{ }^{\ddagger}-\frac{1}{M_{3 / 2}}\left[M_{3 / 2} \gamma^{n}-\sigma^{n k} i \partial_{k}\right] \theta_{\chi}^{\ddagger}, \\
\tilde{\Phi}_{\eta} & =\Phi_{\eta}-\frac{1}{M_{2}} \theta_{\eta}^{0}, \\
\tilde{\Phi}_{2}^{(1)} & =\Phi_{2}^{(1)}+c \theta_{2}^{00}+\frac{1}{2 M^{2}}\left(\frac{1-c}{3+c}\right)\left(2 \partial^{k} \partial_{k}+3 M^{2}\right) \theta_{\epsilon}, \\
\tilde{\theta}_{2}^{0 n} & =\theta_{2}^{0 n}+\frac{1}{(3+c)} \partial^{n} \tilde{\Phi}_{\eta}, \\
\tilde{\Phi}_{2}^{(2)} & =\Phi_{2}^{(2)}+2 \partial_{k} \tilde{\theta}_{2}^{0 k} .
\end{aligned}
$$


With these new constraints the remaining non-vanishing Pb's are

$$
\begin{aligned}
\left\{\theta_{1}^{0}(x), \theta_{B}(y)\right\}_{P} & =-M_{1} \delta^{3}(x-y), \\
\left\{\theta_{3 / 2}^{0}(x), \tilde{\Phi}_{\chi}(y)\right\}_{P} & =\gamma_{0} \delta^{3}(x-y)=-\left\{\theta_{3 / 2}^{0}{ }^{\ddagger}(x), \tilde{\Phi}_{\chi}^{\ddagger}(y)\right\}_{P}, \\
\left\{\theta_{\chi}(x), \Phi_{3 / 2}^{0}(y)\right\}_{P} & =M_{3 / 2} \delta^{3}(x-y)=-\left\{\theta_{\chi}^{\ddagger}(x), \Phi_{3 / 2}^{0}{ }^{\ddagger}(y)\right\}_{P}, \\
\left\{\tilde{\theta}_{3 / 2}^{n}(x), \tilde{\theta}_{3 / 2}^{m \ddagger}(y)\right\}_{P} & =-i \sigma^{m n} \delta^{3}(x-y), \\
\left\{\theta_{2}^{00}(x), \theta_{\eta}^{0}(y)\right\}_{P} & =-M_{2} \delta^{3}(x-y), \\
\left\{\tilde{\theta}_{2}^{0 n}(x), \theta_{\eta}^{m}(y)\right\}_{P} & =-M_{2} g^{n m} \delta^{3}(x-y), \\
\left\{\theta_{\epsilon}(x), \tilde{\Phi}_{2}^{(2)}(y)\right\}_{P} & =2\left(\frac{3+c}{1-c}\right) M_{2}^{2} \delta^{3}(x-y), \\
\left\{\tilde{\Phi}_{2}^{(1)}(x), \tilde{\Phi}_{\eta}(y)\right\}_{P} & =-(3+c) \delta^{3}(x-y) .
\end{aligned}
$$

The $\mathrm{Db}$ and the inverse functions that go with them are defined in (19) and (20), so we can immediately write down the ETC and ETAC relations

$$
\begin{aligned}
& {\left[A^{\mu}(x), \dot{A}^{\nu}(y)\right]_{0}=-i\left(g^{\mu \nu}-(1-a) \delta_{0}^{\mu} \delta_{0}^{\nu}\right) \delta^{3}(x-y),} \\
& {\left[A^{\mu}(x), B(y)\right]_{0}=\frac{i}{M_{1}} \delta_{0}^{\mu} \delta^{3}(x-y),} \\
& {\left[A^{\mu}(x), \dot{B}(y)\right]_{0}=-\left[\dot{A}_{\mu}(x), B(y)\right]_{0}=-i \delta_{k}^{\mu} \frac{\partial^{k}}{M_{1}} \delta^{3}(x-y),} \\
& {[B(x), \dot{B}(y)]_{0}=-i \delta^{3}(x-y),} \\
& \left\{\psi^{n}(x), \psi^{m \dagger}(y)\right\}_{0}=-\left[g^{n m}-\frac{1}{2} \gamma^{n} \gamma^{m}\right] \delta^{3}(x-y), \\
& \left\{\psi^{0}(x), \psi^{0^{\dagger}}(y)\right\}_{0}=-\frac{3}{2}(1+b)^{2} \delta^{3}(x-y), \\
& \left\{\psi^{0}(x), \psi^{m \dagger}(y)\right\}_{0}=\left[\frac{b+1}{2} \gamma^{m}-b \frac{i \partial^{m}}{M_{3 / 2}}\right] \gamma_{0} \delta^{3}(x-y), \\
& \left\{\psi^{n}(x), \psi^{0^{\dagger}}(y)\right\}_{0}=\left[\frac{b+1}{2} \gamma^{n}-b \frac{i \partial^{n}}{M_{3 / 2}}\right] \gamma_{0} \delta^{3}(x-y), \\
& \left\{\chi(x), \chi^{\dagger}(y)\right\}_{0}=-\frac{3}{2} \delta^{3}(x-y), \\
& \left\{\psi^{0}(x), \chi^{\dagger}(y)\right\}_{0}=\gamma_{0}\left[\frac{3(1+b)}{2}-\frac{1}{M_{3 / 2}} i \gamma^{k} \partial_{k}\right] \delta^{3}(x-y), \\
& \left\{\psi^{n}(x), \chi^{\dagger}(y)\right\}_{0}=-\left[\frac{1}{2} \gamma^{n}-\frac{i \partial^{n}}{M_{3 / 2}}\right] \delta^{3}(x-y), \\
& {\left[h^{00}(x), \eta^{0}(y)\right]_{0}=\frac{3}{M_{2}(3+c)} i \delta^{3}(x-y),} \\
& {\left[h^{0 n}(x), \eta^{m}(y)\right]_{0}=\frac{1}{M_{2}} g^{n m} i \delta^{3}(x-y),} \\
& {\left[h^{0 n}(x), \epsilon(y)\right]_{0}=-\frac{1}{M_{2}^{2}}\left(\frac{1-c}{3+c}\right) \partial^{n} i \delta^{3}(x-y),}
\end{aligned}
$$




$$
\begin{aligned}
{\left[h^{n m}(x), \eta^{0}(y)\right]_{0} } & =-\frac{1}{M_{2}(3+c)} g^{n m} i \delta^{3}(x-y), \\
{\left[\eta^{0}(x), \eta^{m}(y)\right]_{0} } & =\frac{1}{M_{2}^{2}(3+c)} \partial^{m} i \delta^{3}(x-y), \\
{\left[\eta^{0}(x), \epsilon(y)\right]_{0} } & =\frac{3}{2 M_{2}} \frac{(1-c)}{(3+c)^{2}} i \delta^{3}(x-y) .
\end{aligned}
$$

In principle there are also ETC relations among time derivatives of the fields in (48), that we have not shown for convenience. However, they are of importance when calculating the commutation relations for non-equal times, below.

\subsection{Propagators}

In order to get commutation and anti-commutation relations for non-equal times we first construct solutions to the EoMs ( (29), (30) and (31)) based on the identities (22)

$$
\begin{aligned}
& B(x)=\int d^{3} z\left[\partial_{0}^{z} \Delta\left(x-z ; M_{B}^{2}\right) \cdot B(z)-\Delta\left(x-z ; M_{B}^{2}\right) \cdot \partial_{0}^{z} B(z)\right], \\
& A_{\mu}(x)=\int d^{3} z\left[\partial_{0}^{z} \Delta\left(x-z ; M_{1}^{2}\right) \cdot A_{\mu}(z)-\Delta\left(x-z ; M_{1}^{2}\right) \cdot \partial_{0}^{z} A_{\mu}(z)\right] \\
& +\frac{1}{(1-a) M_{1}^{2}} \int d^{3} z\left[\left(\partial_{0}^{z} \Delta\left(x-z ; M_{B}^{2}\right)-\partial_{0}^{z} \Delta\left(x-z ; M_{1}^{2}\right)\right)\right. \\
& \left.-\left(\Delta\left(x-z ; M_{B}^{2}\right)-\Delta\left(x-z ; M_{1}^{2}\right)\right) \partial_{0}^{z}\right] \\
& \times\left(\square+M_{1}^{2}\right) A_{\mu}(z), \\
& \chi(x)=i \int d^{3} z\left(i \not \partial_{x}+M_{\chi}\right) \gamma^{0} \Delta\left(x-z ; M_{\chi}^{2}\right) \chi(z), \\
& \psi_{\mu}(x)=i \int d^{3} z\left(i \not \partial_{x}+M_{3 / 2}\right) \gamma^{0} \Delta\left(x-z ; M_{3 / 2}^{2}\right) \psi_{\mu}(z) \\
& +\frac{2 i}{3(b+2) M_{3 / 2}} \int d^{3} z\left[\left(i \not x_{x}+M_{3 / 2}\right) \Delta\left(x-z ; M_{3 / 2}^{2}\right)\right. \\
& \left.-\left(i \not \not_{x}-M_{\chi}\right) \Delta\left(x-z ; M_{\chi}^{2}\right)\right] \gamma^{0}\left(i \not \partial_{z}-M_{3 / 2}\right) \psi_{\mu}(z) \\
& +\frac{2 i}{(3 b+2) M_{3 / 2}} \int d^{3} z\left\{\Delta\left(x-z ; M_{\chi}^{2}\right)-\frac{2}{3(b+2) M_{3 / 2}}[\right. \\
& \left.\left.\times\left(i \not_{x}+M_{3 / 2}\right) \Delta\left(x-z ; M_{3 / 2}^{2}\right)-\left(i \not_{x}-M_{\chi}\right) \Delta\left(x-z ; M_{\chi}^{2}\right)\right]\right\} \\
& \times \gamma^{0}\left(i \not \partial_{z}+M_{\chi}\right)\left(i \not \partial_{z}-M_{3 / 2}\right) \psi_{\mu}(z), \\
& \epsilon(x)=\int d^{3} z\left[\partial_{0}^{z} \Delta\left(x-z ; M_{\epsilon}^{2}\right) \cdot \epsilon(z)-\Delta\left(x-z ; M_{\epsilon}^{2}\right) \cdot \partial_{0}^{z} \epsilon(z)\right], \\
& \eta^{\mu}(x)=\int d^{3} z\left[\partial_{0}^{z} \Delta\left(x-z ; M_{\eta}^{2}\right) \cdot \eta^{\mu}(z)-\Delta\left(x-z ; M_{\eta}^{2}\right) \cdot \partial_{0}^{z} \eta^{\mu}(z)\right] \\
& +\frac{1}{M_{\eta}^{2}-M_{\epsilon}^{2}} \int d^{3} z\left[\partial_{0}^{z}\left(\Delta\left(x-z ; M_{\epsilon}^{2}\right)-\Delta\left(x-z ; M_{\eta}^{2}\right)\right)\right. \\
& \left.-\left(\Delta\left(x-z ; M_{\epsilon}^{2}\right)-\Delta\left(x-z ; M_{\eta}^{2}\right)\right) \cdot \partial_{0}^{z}\right]\left(\square+M_{\eta}^{2}\right) \eta^{\mu}(z),
\end{aligned}
$$




$$
\begin{aligned}
& h^{\mu \nu}(x)= \int d^{3} z\left[\partial_{0}^{z} \Delta\left(x-z ; M_{2}^{2}\right) \cdot h^{\mu \nu}(z)-\Delta\left(x-z ; M_{2}^{2}\right) \cdot \partial_{0}^{z} h^{\mu \nu}(z)\right] \\
&+ \frac{1}{M_{2}^{2}-M_{\eta}^{2}} \int d^{3} z\left[\partial_{0}^{z}\left(\Delta\left(x-z ; M_{\eta}^{2}\right)-\Delta\left(x-z ; M_{2}^{2}\right)\right)\right. \\
&\left.-\left(\Delta\left(x-z ; M_{\eta}^{2}\right)-\Delta\left(x-z ; M_{2}^{2}\right)\right) \partial_{0}^{z}\right] \\
& \times\left(\square+M_{2}^{2}\right) h^{\mu \nu}(z) \\
&+ \frac{1}{\left(M_{\eta}^{2}-M_{\epsilon}^{2}\right)\left(M_{2}^{2}-M_{\eta}^{2}\right)\left(M_{2}^{2}-M_{\epsilon}^{2}\right)} \int d^{3} z[ \\
& \partial_{0}^{z}\left(\left(M_{2}^{2}-M_{\eta}^{2}\right) \Delta\left(x-z ; M_{\epsilon}^{2}\right)-\left(M_{2}^{2}-M_{\epsilon}^{2}\right) \Delta\left(x-z ; M_{\eta}^{2}\right)\right.\left.+\left(M_{\eta}^{2}-M_{\epsilon}^{2}\right) \Delta\left(x-z ; M_{2}^{2}\right)\right) \\
&-\left(\left(M_{2}^{2}-M_{\eta}^{2}\right) \Delta\left(x-z ; M_{\epsilon}^{2}\right)-\left(M_{2}^{2}-M_{\epsilon}^{2}\right) \Delta\left(x-z ; M_{\eta}^{2}\right)\right. \\
&\left.\left.+\left(M_{\eta}^{2}-M_{\epsilon}^{2}\right) \Delta\left(x-z ; M_{2}^{2}\right)\right) \partial_{0}^{z}\right]\left(\square+M_{\eta}^{2}\right)\left(\square+M_{2}^{2}\right) h^{\mu \nu}(z) .
\end{aligned}
$$

Using these equations (49) and the ETC and ETAC relations of (46), (47) and (48) we obtain the following commutation and anti-commutation relations

$$
\begin{aligned}
{[B(x), B(y)]=} & -i \Delta\left(x-y, M_{B}^{2}\right), \\
{\left[A^{\mu}(x), B(y)\right]=} & -i \frac{\partial^{\mu}}{M_{1}} \Delta\left(x-y, M_{B}^{2}\right), \\
{\left[A^{\mu}(x), A^{\nu}(y)\right]=} & -i\left(g^{\mu \nu}+\frac{\partial^{\mu} \partial^{\nu}}{M_{1}^{2}}\right) \Delta\left(x-y ; M_{1}^{2}\right)+i \frac{\partial^{\mu} \partial^{\nu}}{M_{1}^{2}} \Delta\left(x-y ; M_{B}^{2}\right) \\
= & P_{1}^{\mu \nu} i \Delta\left(x-y ; M_{1}^{2}\right)+P_{B}^{\mu \nu} i \Delta\left(x-y ; M_{B}^{2}\right), \\
\{\chi(x), \bar{\chi}(y)\}= & -\frac{3}{2} i\left(i \not \partial+M_{\chi}\right) \Delta\left(x-y ; M_{\chi}^{2}\right), \\
\left\{\psi^{\mu}(x), \bar{\chi}(y)\right\}= & -\frac{1}{2}\left[\gamma^{\mu}-\frac{2 i \partial^{\mu}}{M_{3 / 2}}\right] i\left(i \not \partial+M_{\chi}\right) \Delta\left(x-y ; M_{\chi}^{2}\right), \\
\left\{\psi^{\mu}(x), \bar{\psi}^{\nu}(y)\right\}= & -i\left(i \not \partial+M_{3 / 2}\right)\left[g^{\mu \nu}-\frac{1}{3} \gamma^{\mu} \gamma^{\nu}+\frac{2 \partial^{\mu} \partial^{\nu}}{3 M_{3 / 2}^{2}}\right. \\
& -\frac{1}{6}\left[\gamma^{\mu}-\frac{2 i \partial^{\mu}}{M_{3 / 2}}\right] i\left(i \not \partial+M_{\chi}\right)\left[\gamma^{\nu}-\frac{2 i \partial^{\nu}}{M_{3 / 2}}\right] \Delta\left(x-y ; \lambda_{\chi}^{2}\right) \\
= & \left(i \not \partial+M_{3 / 2}\right) P_{3 / 2}^{\mu \nu} i \Delta\left(x-y ; \gamma_{3 / 2}^{2}\right)+P_{\chi}^{\mu \nu} i \Delta\left(x-y ; M_{\chi}^{2}\right), \\
{[\epsilon(x), \epsilon(y)]=} & -\frac{3}{4} \frac{c(1-c)^{2}}{(3+c)^{3}} i \Delta\left(x-y ; M_{\epsilon}^{2}\right), \\
{\left[\eta^{\mu}(x), \epsilon(y)\right]=} & -\frac{3}{2} \frac{(1-c)}{(3+c)^{2}} \frac{\partial^{\mu}}{M_{2}} i \Delta\left(x-y ; M_{\epsilon}^{2}\right), \\
{\left[\eta^{\mu}(x), \eta^{\nu}(y)\right]=} & {\left[g^{\mu \nu}+\frac{\partial^{\mu} \partial^{\nu}}{M_{\eta}^{2}}\right] i \Delta\left(x-y ; M_{\eta}^{2}\right)-\frac{3}{(3+c)} \frac{\partial^{\mu} \partial^{\nu}}{M_{\eta}^{2}} i \Delta\left(x-y ; M_{\epsilon}^{2}\right), } \\
{\left[\epsilon(x), h^{\mu \nu}(y)\right]=} & \frac{(1-c)}{(3+c)}\left[\frac{\partial^{\mu} \partial^{\nu}}{M_{2}^{2}}-\frac{1}{2} \frac{c}{(3+c)} g^{\mu \nu}\right] i \Delta\left(x-y ; M_{\epsilon}^{2}\right),
\end{aligned}
$$




$$
\begin{aligned}
{\left[\eta^{\alpha}(x), h^{\mu \nu}(y)\right]=} & \frac{1}{M_{2}}\left[\partial^{\mu} g^{\alpha \nu}+\partial^{\nu} g^{\alpha \mu}+\frac{2}{M_{\eta}^{2}} \partial^{\alpha} \partial^{\mu} \partial^{\nu}\right] i \Delta\left(x-y ; M_{\eta}^{2}\right) \\
& -\frac{1}{M_{2}}\left[\frac{1}{(3+c)} \partial^{\alpha} g^{\mu \nu}+\frac{2}{M_{\eta}^{2}} \partial^{\alpha} \partial^{\mu} \partial^{\nu}\right] i \Delta\left(x-y ; M_{\epsilon}^{2}\right) \\
{\left[h^{\mu \nu}(x), h^{\alpha \beta}(y)\right]=} & {\left[g^{\mu \alpha} g^{\nu \beta}+g^{\mu \beta} g^{\nu \alpha}-\frac{2}{3} g^{\mu \nu} g^{\alpha \beta}\right.} \\
& +\frac{1}{M_{2}^{2}}\left(\partial^{\mu} \partial^{\alpha} g^{\nu \beta}+\partial^{\nu} \partial^{\alpha} g^{\mu \beta}+\partial^{\mu} \partial^{\beta} g^{\nu \alpha}+\partial^{\nu} \partial^{\beta} g^{\mu \alpha}\right) \\
& \left.-\frac{2}{3 M_{2}^{2}}\left(\partial^{\mu} \partial^{\nu} g^{\alpha \beta}+g^{\mu \nu} \partial^{\alpha} \partial^{\beta}\right)+\frac{4}{3 M_{2}^{4}} \partial^{\mu} \partial^{\nu} \partial^{\alpha} \partial^{\beta}\right] i \Delta\left(x-y ; M_{2}^{2}\right) \\
& -\frac{1}{M_{2}^{2}}\left[\partial^{\mu} \partial^{\alpha} g^{\nu \beta}+\partial^{\nu} \partial^{\alpha} g^{\mu \beta}+\partial^{\mu} \partial^{\beta} g^{\nu \alpha}+\partial^{\nu} \partial^{\beta} g^{\mu \alpha}\right. \\
& \left.+\frac{4}{M_{\eta}^{2}} \partial^{\mu} \partial^{\nu} \partial^{\alpha} \partial^{\beta}\right] i \Delta\left(x-y ; M_{\eta}^{2}\right) \\
& -\left[\frac{1}{3} \frac{c}{3+c} g^{\mu \nu} g^{\alpha \beta}-\frac{2}{3 M_{2}^{2}}\left(\partial^{\mu} \partial^{\nu} g^{\alpha \beta}+g^{\mu \nu} \partial^{\alpha} \partial^{\beta}\right)\right. \\
& \left.+\frac{4(3+c)}{3 c M_{2}^{4}} \partial^{\mu} \partial^{\nu} \partial^{\alpha} \partial^{\beta}\right] i \Delta\left(x-y ; M_{\epsilon}^{2}\right) \\
= & 2 P_{2}^{\mu \nu \alpha \beta}(\partial) i \Delta\left(x-y ; M_{2}^{2}\right)+P_{\eta}^{\mu \nu \alpha \beta}(\partial) i \Delta\left(x-y ; M_{\eta}^{2}\right) \\
+ & P_{\epsilon}^{\mu \nu \alpha \beta}(\partial) i \Delta\left(x-y ; M_{\epsilon}^{2}\right) .
\end{aligned}
$$

From the overall minus signs in the (anti-) commutation relations of the auxiliary fields in (52) we conclude that all auxiliary fields are ghost, except for the $\epsilon$-field. There the choice of the gauge parameter $c$ determines whether it is ghost-like or not: for $-3<c<0$ the $\epsilon$-field is physical and it ghost-like in all other cases (excluding $c=-3$ and $c=0$ ).

Having obtained these (anti-) commutation relations we calculate the propagators

$$
\begin{aligned}
D_{F, a}^{\mu \nu}(x-y)= & -i<0\left|T\left[A^{\mu}(x), A^{\nu}(y)\right]\right| 0> \\
= & -i \theta\left(x_{0}-y_{0}\right)\left[P_{1}^{\mu \nu}(\partial) \Delta^{(+)}\left(x-y ; M_{1}^{2}\right)+P_{B}^{\mu \nu}(\partial) \Delta^{(+)}\left(x-y ; M_{B}^{2}\right)\right] \\
& -i \theta\left(x_{0}-y_{0}\right)\left[P_{1}^{\mu \nu}(\partial) \Delta^{(-)}\left(x-y ; M_{1}^{2}\right)+P_{B}^{\mu \nu}(\partial) \Delta^{(-)}\left(x-y ; M_{B}^{2}\right)\right] \\
= & P_{1}^{\mu \nu}(\partial) \Delta_{F}\left(x-y ; M_{1}^{2}\right)+P_{B}^{\mu \nu}(\partial) \Delta_{F}\left(x-y ; M_{B}^{2}\right) .
\end{aligned}
$$

We see that this propagator is explicitly covariant, independent of the choice of the gauge parameter. Choosing $a=1$ we see that the terms containing derivatives cancel and that only the $g^{\mu \nu}$ term remains. It can be seen as the massive photon propagator. For $a=\infty$ we re-obtain the massive spin-1 field, like in (24). Except in the above derivation it is obtained without non-covariant terms in the propagator. The choice $a=0$ is particularly interesting, because then still the spin- 1 condition $\partial \cdot A=0$ holds (text below (29) ), but the propagator is covariant. In momentum space it looks like

$$
D_{F, 0}^{\mu \nu}(P)=\frac{-g^{\mu \nu}+\frac{p^{\mu} p^{\nu}}{p^{2}}}{p^{2}-M_{1}^{2}+i \varepsilon} .
$$

The spin-3/2 propagator is

$$
\begin{aligned}
S_{F, b}^{\mu \nu}(x-y)= & -i<0\left|T\left[\psi^{\mu}(x), \bar{\psi}^{\nu}(y)\right]\right| 0> \\
= & -i \theta\left(x_{0}-y_{0}\right)\left[\left(i \not \partial+M_{3 / 2}\right) P_{3 / 2}^{\mu \nu}(\partial) \Delta^{(+)}\left(x-y ; M_{3 / 2}^{2}\right)+P_{\chi}^{\mu \nu}(\partial) \Delta^{(+)}\left(x-y ; M_{\chi}^{2}\right)\right] \\
& -i \theta\left(x_{0}-y_{0}\right)\left[\left(i \not \partial+M_{3 / 2}\right) P_{3 / 2}^{\mu \nu}(\partial) \Delta^{(-)}\left(x-y ; M_{3 / 2}^{2}\right)+P_{\chi}^{\mu \nu}(\partial) \Delta^{(-)}\left(x-y ; M_{\chi}^{2}\right)\right]
\end{aligned}
$$




$$
\begin{aligned}
= & \left(i \not \partial+M_{3 / 2}\right) P_{3 / 2}^{\mu \nu}(\partial) \Delta_{F}\left(x-y ; M_{1}^{2}\right)+P_{\chi}^{\mu \nu}(\partial) \Delta_{F}\left(x-y ; M_{B}^{2}\right) \\
& +\frac{b}{M_{3 / 2}} \delta_{0}^{\mu} \delta_{0}^{\nu} \delta^{4}(x-y) .
\end{aligned}
$$

Only for $b=0$ we have an explicitly covariant propagator. This result was also obtained in [17. From the text below (30) we see that the choice $b=0$ means that we have only one of the two spin- $3 / 2$ conditions or, to put it in a different way, we have added an extra spin- $1 / 2$ piece to make the RS propagator explicitly covariant.

For $b=-\frac{4}{3}$ and $b=-1$ we have that $i \partial \cdot \psi=0($, but $\gamma \cdot \psi \neq 0)$, but then the propagator is not covariant anymore.

The spin-2 propagator is

$$
\begin{aligned}
D_{F, c}^{\mu \nu \alpha \beta}(x-y)= & -i<0\left|T\left[h^{\mu \nu}(x) h^{\alpha \beta}(y)\right]\right| 0> \\
= & -i \theta\left(x^{0}-y^{0}\right)\left[2 P_{2}^{\mu \nu \alpha \beta}(\partial) \Delta^{(+)}\left(x-y ; M^{2}\right)+P_{\eta}^{\mu \nu \alpha \beta}(\partial) i \Delta^{(+)}\left(x-y ; M_{\eta}^{2}\right)\right. \\
& \left.\quad+P_{\epsilon}^{\mu \nu \alpha \beta}(\partial) i \Delta^{(+)}\left(x-y ; M_{\epsilon}^{2}\right)\right] \\
& -i \theta\left(y^{0}-x^{0}\right)\left[2 P_{2}^{\mu \nu \alpha \beta}(\partial) \Delta^{(-)}\left(x-y ; M^{2}\right)+P_{\eta}^{\mu \nu \alpha \beta}(\partial) i \Delta^{(-)}\left(x-y ; M_{\eta}^{2}\right)\right. \\
& \left.\quad+P_{\epsilon}^{\mu \nu \alpha \beta}(\partial) i \Delta^{(-)}\left(x-y ; M_{\epsilon}^{2}\right)\right] \\
= & 2 P_{2}^{\mu \nu \alpha \beta}(\partial) \Delta_{F}\left(x-y ; M^{2}\right)+P_{\eta}^{\mu \nu \alpha \beta}(\partial) \Delta_{F}\left(x-y ; M_{\eta}^{2}\right) \\
& +P_{\epsilon}^{\mu \nu \alpha \beta}(\partial) \Delta_{F}\left(x-y ; M_{\epsilon}^{2}\right) .
\end{aligned}
$$

We see that this propagator (56) does not contain local, non-covariant terms independent of the choice of the gauge parameter. The first part of (56) $\left(P_{2}^{\mu \nu \alpha \beta}(\partial)\right.$-part) is pure spin-2 12 . The nature of the other parts depends on the free gauge parameter.

Since $c$ is still a free parameter it is interesting to look at several gauges. But before that, we exclude $c=1$ and $c=-3$ as before. In these cases the $\epsilon$-field vanishes and the EoM are quite different. Also the quantization procedure runs differently.

An interesting gauge which we want to discuss here is $c=-1$. From (31) we see that all fields become free Klein-Gordon fields of mass $M_{2}$. As a result of this choice all derivative terms disappear in (56) and what is left is

$$
D_{F,-1}^{\mu \nu \alpha \beta}(x-y)=\left[g^{\mu \alpha} g^{\nu \beta}+g^{\mu \beta} g^{\nu \alpha}-\frac{1}{2} g^{\mu \nu} g^{\alpha \beta}\right] \Delta_{F}\left(x-y ; M^{2}\right) .
$$

In contrast to the spin-1 case, discussed above, equation (57) is not the massive version of the massless spin-2 propagator.

Equation (56) yields for the choice $c=0$

$$
\begin{aligned}
D_{F, 0}^{\mu \nu \alpha \beta}(x-y)= & 2 P_{2}^{\mu \nu \alpha \beta}(\partial) \Delta_{F}\left(x-y ; M_{2}^{2}\right)-\frac{1}{M_{2}^{2}}\left[\partial^{\mu} \partial^{\alpha} g^{\nu \beta}+\partial^{\nu} \partial^{\alpha} g^{\mu \beta}+\partial^{\mu} \partial^{\beta} g^{\nu \alpha}+\partial^{\nu} \partial^{\beta} g^{\mu \alpha}\right. \\
& \left.-\frac{2}{3}\left(\partial^{\mu} \partial^{\nu} g^{\alpha \beta}+g^{\mu \nu} \partial^{\alpha} \partial^{\beta}\right)+\frac{4 \partial^{\mu} \partial^{\nu} \partial^{\alpha} \partial^{\beta}}{3 M_{2}^{2}}\right] \Delta_{F}(x-y) \\
& +\frac{4}{3 M_{2}^{2}} \partial^{\mu} \partial^{\nu} \partial^{\alpha} \partial^{\beta} \tilde{\Delta}_{F}(x-y) \\
D_{F, 0}^{\mu \nu \alpha \beta}(p)= & {\left[g^{\mu \alpha} g^{\nu \beta}+g^{\mu \beta} g^{\nu \alpha}-\frac{2}{3} g^{\mu \nu} g^{\alpha \beta}+\frac{2}{3 p^{2}}\left(p^{\mu} p^{\nu} g^{\alpha \beta}+g^{\mu \nu} p^{\alpha} p^{\beta}\right)\right.} \\
& \left.-\frac{1}{p^{2}}\left(p^{\mu} p^{\alpha} g^{\nu \beta}+p^{\nu} p^{\alpha} g^{\mu \beta}+p^{\mu} p^{\beta} g^{\nu \alpha}+p^{\nu} p^{\beta} g^{\mu \alpha}\right)+\frac{4}{3 p^{4}} p^{\mu} p^{\nu} p^{\alpha} p^{\beta}\right] \\
& \times \frac{1}{p^{2}-M_{2}^{2}+i \varepsilon} .
\end{aligned}
$$

\footnotetext{
${ }^{12}$ The factor 2 can again be transformed away by redefining all fields as in 23)
} 
Here, the $\tilde{\Delta}_{F}(x-y)$ (as well as various other $\Delta$ propagators) is defined in appendix A As in the spin1 case this propagator (58) satisfies the field equations (and is therefore pure spin-2) and is explicitly covariant. This result is also obtained by ignoring the $c$ term in the Lagrangian (28a) from the outset.

\subsection{Massless limit}

It is most easy to study the massless limits of the propagators obtained in the previous subsection in momentum space

$$
\operatorname{Lim}_{M_{1} \rightarrow 0} D_{F, a}^{\mu \nu}(p)=\left[-g^{\mu \nu}+(1-a) \frac{p^{\mu} p^{\nu}}{p^{2}}\right] \frac{1}{p^{2}+i \varepsilon}
$$

Although we have not presented the massless case, it is done rather easily. The quantization procedure runs very similar to what is presented in section 3.2 contrary to the case without an auxiliary field (section 2.2), only the equations like in (49) are a bit different. It should be noticed that it is sufficient in the massless case to ignore the mass term of the spin-1 field in (28a), only. So, even though allowing for a mass term for the auxiliary field, both $A^{\mu}$ and $B$ turn out to be massless. Therefore the freedom in choosing the gauge parameter is still present. In the massless case the exact same result as (59) is obtained, so the massless limit connects smoothly with the massless case and is explicitly covariant. In fact this line of reasoning is valid for all three spin cases with auxiliary fields. Having mentioned this, we will not come back to this when discussing the massless limits of the spin- $3 / 2$ and spin- 2 cases below.

The massless limit of the spin- $3 / 2$ field is

$$
\operatorname{Lim}_{M_{3 / 2} \rightarrow 0} S_{F, 0}^{\mu \nu}(p)=-\not p\left[g^{\mu \nu}-\frac{1}{2} \gamma^{\mu} \gamma^{\nu}\right] \frac{1}{p^{2}+i \varepsilon}+\gamma^{\mu} p^{\nu} \frac{1}{p^{2}+i \varepsilon}-2 p^{\mu} p^{\nu} \not p \frac{1}{p^{4}+i \varepsilon} .
$$

We notice that when this propagator (60) is coupled to conserved currents only the first two parts contribute. These parts form exactly the massless spin- $3 / 2$ propagator with only the helicities $\lambda= \pm 3 / 2$ ([26]). When we couple the (massive) RS-propagator (25) to conserved currents and take the massless limit 13 we see that it is different from the one in (60) because of the factor in front of the $\gamma^{\mu} \gamma^{\nu}$ term.

The massless limit of the spin-2 propagator is

$$
\begin{aligned}
\operatorname{Lim}_{M_{2} \rightarrow 0} D_{F, c}^{\mu \nu \alpha \beta}(p)= & {\left[g^{\mu \alpha} g^{\nu \beta}+g^{\mu \beta} g^{\nu \alpha}-\frac{2+c}{3+c} g^{\mu \nu} g^{\alpha \beta}\right] \frac{1}{p^{2}+i \varepsilon} } \\
& \quad-(1+c) \frac{1}{p^{2}}\left[p^{\mu} p^{\alpha} g^{\nu \beta}+p^{\nu} p^{\alpha} g^{\mu \beta}+p^{\mu} p^{\beta} g^{\nu \alpha}+p^{\nu} p^{\beta} g^{\mu \alpha}\right. \\
& \left.\quad-\frac{2}{3+c}\left(p^{\mu} p^{\nu} g^{\alpha \beta}+g^{\mu \nu} p^{\alpha} p^{\beta}\right)\right] \frac{1}{p^{2}+i \varepsilon} \\
& +\frac{4(1+c)^{2}}{3+c} \frac{p^{\mu} p^{\nu} p^{\alpha} p^{\beta}}{p^{4}} \frac{1}{p^{2}+i \varepsilon}
\end{aligned}
$$

Making the choice of the gauge parameter $c \rightarrow \pm \infty$ we see that (61) becomes the massless spin-2 propagator plus terms proportional to $p$. In physical processes these terms do not contribute when coupled to conserved currents

$$
D_{F, \pm \infty}^{\mu \nu \alpha \beta}(p)=\left[g^{\mu \alpha} g^{\nu \beta}+g^{\mu \beta} g^{\nu \alpha}-g^{\mu \nu} g^{\alpha \beta}\right] \frac{1}{p^{2}+i \varepsilon}+O(p) .
$$

Again, this is different from taking the massive spin-2 propagator (26), couple it to conserved currents and taking the massless limit, as is mentioned in [27.

Having obtained the correct massless spin-2 propagator (61) it is particularly interesting to see how this limit comes about. Considering the propagator (56) (coupled to conserved currents) with a small non-zero mass and requiring that it is a mixture of pure spin-2 and spin-0 (so no ghosts or tachyons) in

\footnotetext{
${ }^{13}$ Terms in the massive RS propagator that do not have a proper massless limit do not contribute since we couple to conserved currents
} 
order to have a kind of massive Brans-Dicke 44 theory, this would imply that $-3<c<0$. However with this restriction we cannot take the mass smoothly to zero in order to have a pure massless spin- 2 propagator, because this requires $c \rightarrow \pm \infty$ as mentioned before.

The above situation of a pure massive spin- 2 and spin-0 propagator limiting smoothly to a pure massless spin-2 propagator can be obtained in [18, but there the set-up is quite different as well as the original goal.

\subsection{Momentum Representation}

To finalize the description of the higher spin fields coupled to auxiliary fields we give the momentum representation of these fields in this subsection. Also, we give the relations which hold for the various creation and annihilation operators.

A solution to the EoM of the fields in (29), (30) and (31) in terms of the auxiliary fields is

$$
\begin{aligned}
A_{\mu} & =V_{\mu}+\frac{\partial_{\mu}}{M_{1}} B \\
\psi_{\mu} & =\Psi_{\mu}+\frac{1}{3}\left(\gamma_{\mu}-\frac{2 i \partial_{\mu}}{M_{3 / 2}}\right) \chi \\
\eta_{\mu} & =\Phi_{1, \mu}+\frac{2(3+c)}{c(1-c)} \frac{\partial_{\mu}}{M_{2}} \epsilon \\
h_{\mu \nu} & =\Phi_{2, \mu \nu}-\frac{1}{M_{2}}\left(\partial_{\mu} \Phi_{1, \nu}+\partial_{\nu} \Phi_{1, \mu}\right)+\frac{2}{3} \frac{3+c}{1-c}\left(g_{\mu \nu}-\frac{2(3+c)}{c} \frac{\partial_{\mu} \partial_{\nu}}{M_{2}^{2}}\right) \epsilon
\end{aligned}
$$

where

$$
\begin{array}{rlrl}
\left(\square+M_{1}^{2}\right) V_{\mu}=0 & , & \partial \cdot V=0 \quad, & \\
\left(i \not \partial-M_{3 / 2}\right) \Psi_{\mu}=0 & , & \gamma \cdot \Psi=0 \quad, & i \partial \cdot \Psi=0, \\
\left(\square+M_{2}^{2}\right) \Phi_{2, \mu \nu}=0 \quad, & \partial^{\mu} \Phi_{2, \mu \nu}=0 \quad, & \Phi_{2, \mu}^{\mu}=0,
\end{array}
$$

and are therefore free spin-1, spin-3/2 and spin-2 fields, respectively. The field $\Phi_{1, \mu}$ also satisfies the free spin-1 equations, but is of negative norm as we will see below.

Since the anti-commutator of the $\chi$-field (51) and the commutator of the $\epsilon$-field (52) contain constants we redefine these fields for convenience

$$
\begin{aligned}
\chi & =\sqrt{\frac{3}{2}} \chi^{\prime} \\
\epsilon & =\frac{\sqrt{3}(1-c)}{2(3+c)} \epsilon^{\prime}
\end{aligned}
$$

14 Therefore (63) becomes

$$
\begin{aligned}
\psi_{\mu} & =\Psi_{\mu}+\frac{1}{\sqrt{6}}\left(\gamma_{\mu}-\frac{2 i \partial_{\mu}}{M_{3 / 2}}\right) \chi^{\prime}, \\
\eta_{\mu} & =\Phi_{1, \mu}+\frac{\sqrt{3}}{c} \frac{\partial_{\mu}}{M_{2}} \epsilon^{\prime} \\
h_{\mu \nu} & =\Phi_{2, \mu \nu}-\frac{1}{M_{2}}\left(\partial_{\mu} \Phi_{1, \nu}+\partial_{\nu} \Phi_{1, \mu}\right)+\frac{1}{\sqrt{3}}\left(g_{\mu \nu}-\frac{2(3+c)}{c} \frac{\partial_{\mu} \partial_{\nu}}{M_{2}^{2}}\right) \epsilon^{\prime} .
\end{aligned}
$$

The momentum representation of the fields is

$$
B(x)=\int \frac{d^{3} p}{(2 \pi)^{3} 2 E_{B}}\left[a_{B}(p) e^{-i p x}+a_{B}^{\dagger}(p) e^{i p x}\right]_{p^{0}=E_{B}},
$$

${ }^{14}$ The part in the commutator of the $\epsilon$-field that determines whether $\epsilon$ is ghost-like or not is not taken in the redefinition. 


$$
\begin{gathered}
V_{\mu}(x)=\sum_{\lambda=-1}^{1} \int \frac{d^{3} p}{(2 \pi)^{3} 2 E_{V}}\left[a_{V, \mu}(p \lambda) e^{-i p x}+a_{V, \mu}^{\dagger}(p \lambda) e^{i p x}\right]_{p^{0}=E_{V}}, \\
\chi^{\prime}(x)=\sum_{s=-\frac{1}{2}}^{\frac{1}{2}} \int \frac{d^{3} p}{(2 \pi)^{3} 2 E_{\chi}}\left[b_{\chi}(p s) u_{\chi}(p s) e^{-i p x}+d_{\chi}^{\dagger}(p s) v_{\chi}(p s) e^{i p x}\right]_{p^{0}=E_{\chi}}, \\
\Psi_{\mu}(x)=\sum_{s=-\frac{3}{2}}^{\frac{3}{2}} \int \frac{d^{3} p}{(2 \pi)^{3} 2 E_{\Psi}}\left[b_{\Psi}(p s) u_{\mu}(p s) e^{-i p x}+d_{\Psi}^{\dagger}(p s) v_{\mu}(p s) e^{i p x}\right]_{p^{0}=E_{\Psi}}, \\
\epsilon^{\prime}(x)=\int_{1, \mu}(x)=\sum_{\lambda=-1}^{1} \int \frac{d^{3} p}{(2 \pi)^{3} 2 E_{\epsilon}}\left[a_{\epsilon}(p) e^{-i p x}+a_{\epsilon}^{\dagger}(p) e^{i p x}\right]_{p^{0}=E_{\epsilon}}, \\
\Phi_{2, \mu \nu}=\sum_{\lambda=-2}^{2} \int \frac{d^{3} p}{(2 \pi)^{3} 2 E_{2}}\left[a_{2, \mu \nu}(p \lambda) e^{-i p x}+a_{1, \mu}^{\dagger}(p \lambda) e^{i p x}\right]_{p^{0}=E_{1}},
\end{gathered}
$$

where $E_{i}=\sqrt{|\vec{p}|^{2}+M_{i}^{2}}$. In (67) the spin-3/2 spinor $u_{\mu}(p s)$ is a tensor product of a spin-1 polarization vector and a spin- $1 / 2$ spinor: $u_{\mu}=\epsilon_{\mu} \otimes u$. The normalization of this (spin-1/2) spinor, as well as that of $u_{\chi}$, is $\bar{u}(p s) u\left(p s^{\prime}\right)=2 M \delta_{s s^{\prime}}$ and of course something similar for the $v$-spinors. With this normalization the creation and annihilation operators satisfy the following (commutation) relations

$$
\begin{aligned}
{\left[a_{B}(p), a_{B}^{\dagger}\left(p^{\prime}\right)\right]=} & -(2 \pi)^{3} 2 E_{B} \delta^{3}\left(p-p^{\prime}\right), \\
{\left[a_{V, \mu}(p \lambda), a_{V, \nu}^{\dagger}\left(p^{\prime} \lambda^{\prime}\right)\right]=} & \left(-g_{\mu \nu}+\frac{p_{\mu} p_{\nu}}{M_{1}^{2}}\right)(2 \pi)^{3} 2 E_{V} \delta^{3}\left(p-p^{\prime}\right) \delta_{\lambda \lambda^{\prime}}, \\
\left\{b_{\chi}(p s), b_{\chi}^{\dagger}\left(p^{\prime} s^{\prime}\right)\right\}= & \left\{d_{\chi}(p s), d_{\chi}^{\dagger}\left(p^{\prime} s^{\prime}\right)\right\}=-(2 \pi)^{3} 2 E_{\chi} \delta^{3}\left(p-p^{\prime}\right) \delta_{s s^{\prime}}, \\
\left\{b_{\Psi}(p s), b_{\Psi}^{\dagger}\left(p^{\prime} s^{\prime}\right)\right\}= & \left\{d_{\Psi}(p s), d_{\Psi}^{\dagger}\left(p^{\prime} s^{\prime}\right)\right\}=(2 \pi)^{3} 2 E_{\Psi} \delta^{3}\left(p-p^{\prime}\right) \delta_{s s^{\prime}}, \\
{\left[a_{\epsilon}(p), a_{\epsilon}^{\dagger}\left(p^{\prime}\right)\right]=} & -\frac{c}{3+c}(2 \pi)^{3} 2 E_{\epsilon} \delta^{3}\left(p-p^{\prime}\right), \\
{\left[a_{1, \mu}(p \lambda), a_{1, \nu}^{\dagger}\left(p^{\prime} \lambda^{\prime}\right)\right]=} & -\left(-g_{\mu \nu}+\frac{p_{\mu} p_{\nu}}{M_{\eta}^{2}}\right)(2 \pi)^{3} 2 E_{1} \delta^{3}\left(p-p^{\prime}\right) \delta_{\lambda \lambda^{\prime}}, \\
{\left[a_{2, \mu \nu}(p \lambda), a_{2, \alpha \beta}\left(p^{\prime} \lambda^{\prime}\right)\right]=} & {\left[g_{\mu \alpha} g_{\nu \beta}+g_{\mu \beta} g_{\nu \alpha}-\frac{2}{3} g_{\mu \nu} g_{\alpha \beta}\right.} \\
& -\frac{1}{M_{2}^{2}}\left(p_{\mu} p_{\alpha} g_{\nu \beta}+p_{\nu} p_{\alpha} g_{\mu \beta}+p_{\mu} p_{\beta} g_{\nu \alpha}+p_{\nu} p_{\beta} g_{\mu \alpha}\right) \\
& \left.+\frac{2}{3 M_{2}^{2}}\left(p_{\mu} p_{\nu} g_{\alpha \beta}+g_{\mu \nu} p_{\alpha} p_{\beta}\right)+\frac{4}{3 M_{2}^{4}} p_{\mu} p_{\nu} p_{\alpha} p_{\beta}\right] \\
& \times(2 \pi)^{3} 2 E_{2} \delta^{3}\left(p-p^{\prime}\right) \delta_{\lambda \lambda^{\prime}} .
\end{aligned}
$$

All other (anti-) commutation relations vanish. These (anti-) commutation relations are such that the relations in (50), (51) and (52) remain valid. 
To complete the properties of the fields in momentum space there still are the following relations

$$
\begin{aligned}
p^{\mu} a_{V, \mu}(p \lambda) & =0, \\
p^{\mu} u_{\mu}(p s) & =0, \quad \gamma^{\mu} u_{\mu}(p s)=0, \\
p^{\mu} a_{1, \mu}(p \lambda) & =0, \\
p^{\mu} a_{2, \mu \nu}(p \lambda) & =0, \quad a_{2, \mu}^{\mu}(p \lambda)=0 .
\end{aligned}
$$

\section{Conclusion and Discussion}

We conclude this article by stating that we have quantized (massive) higher spin $(1 \leq j \leq 2)$ fields in both the case where they are free (section 2) and where they are coupled to (an) auxiliary field(s) (section 3). We have presented a full constraint analysis and quantization procedure to come to equal time (anti) commutation relations.

In the free case we have explicitly shown that the constructed propagators are non-covariant, which is well known. In the coupled case, i.e. auxiliary fields are coupled to gauge conditions of the free case, the propagators can be covariant. Only in the spin- $3 / 2$ case this requires a choice of the parameter, namely $b=0$. The obtained propagators have a smooth massless limit and connect perfectly to propagators which would be obtained in the massless case (including (an) auxiliary field(s)).

When coupled to conserved currents we see that it is possible to obtain the correct massless spin- $j$ propagators carrying only the helicities $\lambda= \pm j_{z}$. Only in the spin-3/2 and in the spin- 2 case we have to make choices for the parameters, namely $b=0$ and $c= \pm \infty$. As far as these two cases is concerned, it is a different situation then taking the massive propagator, couple it to conserved currents and putting the mass to zero. We stress that in these cases the limits are only smooth if the massive propagators contain ghost parts.

\section{Appendices}

\section{A $\Delta$ Propagators}

A few definitions of on mass-shell propagators, according to [43], are

$$
\begin{aligned}
\Delta\left(x ; m^{2}\right) & =\frac{-i}{(2 \pi)^{3}} \int d^{4} p \epsilon\left(p_{0}\right) \delta\left(p^{2}-m^{2}\right) e^{-i p x}, \\
\Delta^{ \pm}\left(x ; m^{2}\right) & =(2 \pi)^{-3} \int d^{4} p \theta\left( \pm p_{0}\right) \delta\left(p^{2}-m^{2}\right) e^{-i p x}, \\
\Delta^{(1)}\left(x ; m^{2}\right) & =\frac{1}{(2 \pi)^{3}} \int d^{4} p \delta\left(p^{2}-m^{2}\right) e^{-i p x},
\end{aligned}
$$

which satisfy the relations amongst each other

$$
\begin{aligned}
i \Delta\left(x ; m^{2}\right) & =\Delta^{+}\left(x ; m^{2}\right)-\Delta^{-}\left(x ; m^{2}\right), \\
\Delta^{+}\left(-x ; m^{2}\right) & =\Delta^{-}\left(x ; m^{2}\right), \\
\Delta^{(1)}\left(x ; m^{2}\right) & =\Delta^{+}\left(x ; m^{2}\right)+\Delta^{-}\left(x ; m^{2}\right) .
\end{aligned}
$$

Furthermore, there are the following Green functions

$$
\begin{aligned}
-\Delta_{F}\left(x ; m^{2}\right) & =i\left[\theta\left(x_{0}\right) \Delta^{+}\left(x ; m^{2}\right)+\theta\left(-x_{0}\right) \Delta^{-}\left(x ; m^{2}\right)\right], \\
\Delta_{r e t}\left(x ; m^{2}\right) & =-\theta\left(x^{0}\right) \Delta\left(x ; m^{2}\right),
\end{aligned}
$$




$$
\begin{aligned}
\Delta_{a d v}\left(x ; m^{2}\right) & =\theta\left(-x^{0}\right) \Delta\left(x ; m^{2}\right), \\
\bar{\Delta}\left(x ; m^{2}\right) & =-\frac{1}{2} \epsilon(x-y) \Delta\left(x ; m^{2}\right),
\end{aligned}
$$

where the Green function of the last line of (72) is defined in the book of Nakanishi and Ojima (see [16]). A well known form the the Feynman propagator $\Delta_{F}(x-y)$ is

$$
\Delta_{F}\left(x ; m^{2}\right)=\frac{1}{(2 \pi)^{4}} \int d^{4} p \frac{e^{-i p x}}{p^{2}-m^{2}+i \varepsilon} .
$$

The following $\Delta$ propagators are defined to be

$$
\begin{aligned}
& \tilde{\Delta}(x)=-\left.\frac{\partial}{\partial m^{2}} \Delta\left(x ; m^{2}\right)\right|_{m^{2}=0}, \\
& \tilde{\tilde{\Delta}}(x)=\left.\left(\frac{\partial}{\partial m^{2}}\right)^{2} \Delta\left(x ; m^{2}\right)\right|_{m^{2}=0} .
\end{aligned}
$$

Since the last two lines of (74) are also valid for Feynman function we can, by using the integral representation of the Feynman function (172) give integral representations for $\tilde{\Delta}_{F}(x)$ and $\tilde{\tilde{\Delta}}_{F}(x)$

$$
\begin{aligned}
& \tilde{\Delta}_{F}\left(x ; m^{2}\right)=-\frac{1}{(2 \pi)^{4}} \int d^{4} p \frac{e^{-i p x}}{p^{4}+i \varepsilon}, \\
& \tilde{\tilde{\Delta}}_{F}\left(x ; m^{2}\right)=\frac{1}{(2 \pi)^{4}} \int d^{4} p \frac{e^{-i p x}}{p^{6}+i \varepsilon} .
\end{aligned}
$$

Furthermore we have the important relations

$$
\begin{gathered}
\left(\square+m^{2}\right) \Delta\left(x ; m^{2}\right)=0, \\
\left.\Delta\left(x ; m^{2}\right)\right|_{0}=0, \\
{\left.\left[\partial_{0} \Delta\left(x ; m^{2}\right)\right]\right|_{0}=-\delta(\vec{x}),} \\
\square \tilde{\Delta}(x)=\Delta(x), \\
\left.\tilde{\Delta}(x)\right|_{0}=\left.\partial_{0} \tilde{\Delta}(x)\right|_{0}=\left.\partial_{0}^{2} \tilde{\Delta}(x)\right|_{0}=0, \\
\left.\partial_{0}^{3} \tilde{\Delta}(x)\right|_{0}=-\delta(\vec{x}), \\
\square \tilde{\tilde{\Delta}}(x)=\tilde{\Delta}(x), \\
\left.\tilde{\tilde{\Delta}}(x)\right|_{0}=\left.\partial_{0} \tilde{\tilde{\Delta}}(x)\right|_{0}=\ldots=\left.\partial_{0}^{4} \tilde{\tilde{\Delta}}(x)\right|_{0}=0, \\
\left.\partial_{0}^{5} \tilde{\tilde{\Delta}}(x)\right|_{0}=-\delta(\vec{x}), \\
{\left.\left[\partial_{0} \Delta^{(1)}\left(x ; m^{2}\right)\right]\right|_{0}=0 .}
\end{gathered}
$$

\section{References}

[1] M.Fierz \& W.Pauli, Proc. Roy. Soc., A173, 211 (1939)

[2] W.Rarita \& J.Schwinger, Phys. Rev. 60, 61 (1941)

[3] P.van Nieuwenhuizen, Phys. Rept. 68, 189 (1981)

[4] K.Johnson \& E.C.G.Sudarshan, Ann. Phys. (N.Y.) 13, 126 (1961)

[5] G.Velo \& D.Zwanziger, Phys. Rev. 186, 267 (1969) 
[6] V.Pascalutsa \& R.Timmermans, Phys. Rev. C60, 042201 (1999)

[7] P.A.M.Dirac, Can. J. Math. 2, 129 (1950); "Lectures on Quantum Mechanics", Yeshiva University, N.Y., 1964; P.A.M.Dirac, Proc. Roy. Soc. Lond. A246, 326 (1958)

[8] G.Senjanovic, Phys. Rev. D16, 307 (1977)

[9] N.S.Baaklini \& M.Tuite, J. Phys. A: Math. Gen. 11, L139 (1978)

[10] V.Pascalutsa, Phys. Rev. D58, 096002 (1998)

[11] V.Pascalutsa, Hadronic J. Suppl. 16, 1 (2001)

[12] R.M.Ghalati, hep-th/0703268 (2007), unpublished

[13] K.R.Green, N.Kiriushcheva \& S.V.Kuzmin, arXiv:0710.1430 [gr-qc] (2008), unpublished

[14] N.S.Baaklini \& M.Tuite, J. Phys. A: Math. Gen. 12, L13 (1978)

[15] S.Weinberg, Phys. Rev 133, B1318 (1964)

[16] N.Nakanishi, Phys. Rev. D5, 1324 (1972), N.Nakanishi and I.Ojima, "Covariant Operator Formalism of Gauge Theories and Quantum Gravity", 1990, World Scientific. Section 2.4.2. This last reference allows for a gauge parameter $\alpha$ as compared to the first one.

[17] K.Babu Joseph \& M.Sabir, J. Phys. A: Math. Gen. 10, 1225 (1977)

[18] T.Kimura, Prog. Theor. Phys. 60, 1940 (1978)

[19] R.Endo and T.Kimura, Prog. Theor. Phys. 61, 1211 (1979)

[20] H.Munczek, Phys. Rev. 164, 1794 (1967)

[21] T.Kimura, Prog. Theor. Phys. 55, 1259 (1976)

[22] N.Nakanishi, Prog. Theor. Phys. 59, 972 (1978)

[23] K.Babu Joseph \& M.Sabir, J. Phys. A: Math. Gen. 13, 2489 (1980)

[24] J.Gomis \& K.Rafanelli, Phys. Rev. D35, 591 (1987)

[25] S.M.Klishevich, Theor. and Math. Phys. 116, 933 (1998)

[26] S.Deser, J.H.Kay and K.S.Stelle, Phys. Rev. D16, 2448 (1977)

[27] H.van Dam \& M.Veltman, Nucl. Phys. B22, 397 (1970), V.I.Zakharov, JETP Lett. 12, 312 (1970)

[28] M.Porrati, Phys. Lett. B498, 92 (2001)

[29] I.I.Kogan, S.Mouslopoulos \& A.Papazoglou, Phys. Lett. B503, 173 (2001)

[30] M.J.Duff, J.T.Liu \& H.Sati, Nucl. Phys. B680, 117 (2004)

[31] P.A.Moldauer \& K.M.Case, Phys. Rev. 102, 279 (1956)

[32] A.Aurillia \& H.Umezawa, Phys. Rev. 182, 1682 (1969)

[33] L.M.Nath, B.Etemadi \& J.D.Kimel, Phys. Rev. D3, 2153 (1971)

[34] M.Benmerrouche, R.M.Davidson \& Nimai.C.Mukhopadhya, Phys. Rev. C39, 2339 (1989)

[35] L.M.Nath, Nucl. Phys. 68, 660 (1965)

[36] S.C.Bhargava \& H.Watanabe, Nucl. Phys. 87, 273 (1966)

[37] A.J.MacFarlane \& W.Tait, Commun. Math. Phys. 24, 211 (1972)

[38] J.Schwinger, "Particles, Sources and Fields", Addison-Wesley Publishing Company, Inc., 1970

[39] A.Salam \& J.Strathdee, Phys. Rev. D14, 2830 (1976)

[40] M.Leclerc, gr-qc/0612125 (2006), unpublished

[41] R.d'Inverno, "Introducing Einstein's relativity", Oxford University Press, 1992

[42] K.Hiida, T.Kimura, T.Ohta and J.Arafune, Prog. Theor. Phys. 45, 1281 (1971)

[43] Björken and Drell, "Relativistic Quatum Fields", McGraw-Hill Publishing Company, 1965

[44] C.Brans \& R.H.Dicke, Phys. Rev. 124, 925 (1961) 